\title{
The spectral function of a first order elliptic system
}

\author{
Olga Chervova, Robert J. Downes, and Dmitri Vassiliev
}

\begin{abstract}
We consider an elliptic self-adjoint first order pseudodifferential operator acting on columns of complex-valued half-densities over a connected compact manifold without boundary. The eigenvalues of the principal symbol are assumed to be simple but no assumptions are made on their sign, so the operator is not necessarily semi-bounded. We study the following objects: the propagator (time-dependent operator which solves the Cauchy problem for the dynamic equation), the spectral function (sum of squares of Euclidean norms of eigenfunctions evaluated at a given point of the manifold, with summation carried out over all eigenvalues between zero and a positive $\lambda$ ), and the counting function (number of eigenvalues between zero and a positive $\lambda$ ). We derive explicit two-term asymptotic formulae for all three. For the propagator "asymptotic" is understood as asymptotic in terms of smoothness, whereas for the spectral and counting functions "asymptotic" is understood as asymptotic with respect to $\lambda \rightarrow+\infty$.
\end{abstract}

Mathematics Subject Classification (2010). Primary 35P20; Secondary 35J46, 35R01.

Keywords. Spectral theory, asymptotic distribution of eigenvalues.

\section{Contents}

1 Main results . . . . . . . . . . . . . . . . . . . . . . . 318

2 Algorithm for the construction of the propagator . . . . . . . . . . . 325

3 Leading transport equations . . . . . . . . . . . . . . . . . 332

4 Proof of formula $(1.20) \ldots \ldots . \ldots 338$

$5 \mathrm{U}(1)$ connection . . . . . . . . . . . . . . . . . . 344

6 Singularity of the propagator at $t=0 \ldots \ldots$. . . . . . . . 347

7 Mollified spectral asymptotics . . . . . . . . . . . . . . . 350

8 Unmollified spectral asymptotics . . . . . . . . . . . . . . . . . 352

$9 \mathrm{U}(m)$ invariance . . . . . . . . . . . . . . . . . . . . . 355

10 Spectral asymmetry . . . . . . . . . . . . . . . . 357

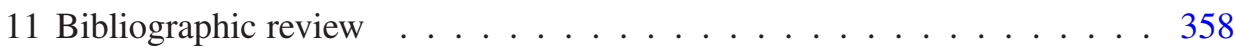

References ...................... . . 359 


\section{Main results}

The aim of the paper is to extend the classical results of [5] to systems. We are motivated by the observation that, to our knowledge, all previous publications on systems give formulae for the second asymptotic coefficient that are either incorrect or incomplete (i.e. an algorithm for the calculation of the second asymptotic coefficient rather than an actual formula). The appropriate bibliographic review is presented in Section 11.

Consider a first order classical pseudodifferential operator $A$ acting on columns $v=\left(\begin{array}{lll}v_{1} & \ldots & v_{m}\end{array}\right)^{T}$ of complex-valued half-densities over a connected compact $n$-dimensional manifold $M$ without boundary. Throughout this paper we assume that $m, n \geq 2$.

We assume the symbol of the operator $A$ to be infinitely smooth. We also assume that the operator $A$ is formally self-adjoint (symmetric):

$$
\int_{M} w^{*} A v d x=\int_{M}(A w)^{*} v d x
$$

for all infinitely smooth $v, w: M \rightarrow \mathbb{C}^{m}$. Here and further on the superscript ${ }^{*}$ in matrices, rows and columns indicates Hermitian conjugation in $\mathbb{C}^{m}$ and

$$
d x \stackrel{\text { def }}{=} d x^{1} \ldots d x^{n}
$$

where $x=\left(x^{1}, \ldots, x^{n}\right)$ are local coordinates on $M$.

Let $A_{1}(x, \xi)$ be the principal symbol of the operator $A$. Here $\xi=\left(\xi_{1}, \ldots, \xi_{n}\right)$ is the variable dual to the position variable $x$; in physics literature the $\xi$ would be referred to as momentum. Our principal symbol $A_{1}$ is an $m \times m$ Hermitian matrix-function on

$$
T^{\prime} M \stackrel{\text { def }}{=} T^{*} M \backslash\{\xi=0\},
$$

i.e. on the cotangent bundle with the zero section removed.

Let $h^{(j)}(x, \xi)$ be the eigenvalues of the principal symbol. We assume these eigenvalues to be nonzero (this is a version of the ellipticity condition) but do not make any assumptions on their sign. We also assume that the eigenvalues $h^{(j)}(x, \xi)$ are simple for all $(x, \xi) \in T^{\prime} M$. The techniques developed in our paper do not work in the case when eigenvalues of the principal symbol have variable multiplicity, though they could probably be adapted to the case of constant multiplicity different from multiplicity 1 . The use of the letter " $h$ " for an eigenvalue of the principal symbol is motivated by the fact that later it will take on the role of a Hamiltonian, see formula (1.13).

We enumerate the eigenvalues of the principal symbol $h^{(j)}(x, \xi)$ in increasing order, using a positive index $j=1, \ldots, m^{+}$for positive $h^{(j)}(x, \xi)$ and a negative index $j=-1, \ldots,-m^{-}$for negative $h^{(j)}(x, \xi)$. Here $m^{+}$is the number of positive eigenvalues of the principal symbol and $m^{-}$is the number of negative ones. Of course, $m^{+}+m^{-}=m$. 
Under the above assumptions $A$ is a self-adjoint operator, in the full functional analytic sense, in the Hilbert space $L^{2}\left(M ; \mathbb{C}^{m}\right)$ (Hilbert space of square integrable complex-valued column "functions") with domain $H^{1}\left(M ; \mathbb{C}^{m}\right)$ (Sobolev space of complex-valued column "functions" which are square integrable together with their first partial derivatives) and the spectrum of $A$ is discrete. These facts are easily established by constructing the parametrix (approximate inverse) of the operator $A+i I$.

Let $\lambda_{k}$ and $v_{k}=\left(v_{k 1}(x) \ldots v_{k m}(x)\right)^{T}$ be the eigenvalues and eigenfunctions of the operator $A$. The eigenvalues $\lambda_{k}$ are enumerated in increasing order with account of multiplicity, using a positive index $k=1,2, \ldots$ for positive $\lambda_{k}$ and a nonpositive index $k=0,-1,-2, \ldots$ for nonpositive $\lambda_{k}$. If the operator $A$ is bounded from below (i.e. if $m^{-}=0$ ) then the index $k$ runs from some integer value to $+\infty$; if the operator $A$ is bounded from above (i.e. if $m^{+}=0$ ) then the index $k$ runs from $-\infty$ to some integer value; and if the operator $A$ is unbounded from above and from below (i.e. if $m^{+} \neq 0$ and $m^{-} \neq 0$ ) then the index $k$ runs from $-\infty$ to $+\infty$.

We will be studying the following three objects.

Object 1. Our first object of study is the propagator, which is the one-parameter family of operators defined as

$$
U(t) \stackrel{\text { def }}{=} e^{-i t A}=\sum_{k} e^{-i t \lambda_{k}} v_{k}(x) \int_{M}\left[v_{k}(y)\right]^{*}(\cdot) d y, \quad t \in \mathbb{R} .
$$

The propagator provides a solution to the Cauchy problem

$$
\left.w\right|_{t=0}=v
$$

for the dynamic equation

$$
D_{t} w+A w=0
$$

where

$$
D_{t} \stackrel{\text { def }}{=}-i \frac{\partial}{\partial t} .
$$

Namely, it is easy to see that if the column of half-densities $v=v(x)$ is infinitely smooth, then, setting

$$
w \stackrel{\text { def }}{=} U(t) v
$$

we get a time-dependent column of half-densities $w(t, x)$ which is also infinitely smooth and which satisfies the equation (1.3) and the initial condition (1.2). The use of the letter " $U$ " for the propagator is motivated by the fact that for each $t$ the operator $U(t)$ is unitary. 
Object 2. Our second object of study is the spectral function, which is the real density defined as

$$
e(\lambda, x, x) \stackrel{\text { def }}{=} \sum_{0<\lambda_{k}<\lambda}\left\|v_{k}(x)\right\|^{2},
$$

where $\left\|v_{k}(x)\right\|^{2} \stackrel{\text { def }}{=}\left[v_{k}(x)\right]^{*} v_{k}(x)$ is the square of the Euclidean norm of the eigenfunction $v_{k}$ evaluated at the point $x \in M$ and $\lambda$ is a positive parameter (spectral parameter).

Object 3. Our third and final object of study is the counting function

$$
N(\lambda) \stackrel{\text { def }}{=} \sum_{0<\lambda_{k}<\lambda} 1=\int_{M} e(\lambda, x, x) d x .
$$

In other words, $N(\lambda)$ is the number of eigenvalues $\lambda_{k}$ between zero and $\lambda$.

It is natural to ask the question: why, in defining the spectral function (1.4) and the counting function (1.5), did we choose to perform summation over all positive eigenvalues up to a given positive $\lambda$ rather than over all negative eigenvalues up to a given negative $\lambda$ ? There is no particular reason. One case reduces to the other by the change of operator $A \mapsto-A$. This issue will be revisited in Section 10.

Further on we assume that $m^{+}>0$, i.e. that the operator $A$ is unbounded from above.

Our objectives are as follows.

Objective 1. We aim to construct the propagator (1.1) explicitly in terms of oscillatory integrals, modulo an integral operator with an infinitely smooth, in the variables $t, x$ and $y$, integral kernel.

Objectives 2 and 3. We aim to derive, under appropriate assumptions on Hamiltonian trajectories, two-term asymptotics for the spectral function (1.4) and the counting function (1.5), i.e. formulae of the type

$$
e(\lambda, x, x)=a(x) \lambda^{n}+b(x) \lambda^{n-1}+o\left(\lambda^{n-1}\right),
$$

and

$$
N(\lambda)=a \lambda^{n}+b \lambda^{n-1}+o\left(\lambda^{n-1}\right)
$$

as $\lambda \rightarrow+\infty$. Obviously, here we expect the real constants $a, b$ and real densities $a(x), b(x)$ to be related in accordance with

$$
a=\int_{M} a(x) d x,
$$


and

$$
b=\int_{M} b(x) d x .
$$

It is well known that the above three objectives are closely related: if one achieves Objective 1, then Objectives 2 and 3 follow via Fourier Tauberian theorems [5], [18], [9], and [17].

We are now in a position to state our main results.

Result 1. We construct the propagator as a sum of $m$ oscillatory integrals

$$
U(t) \stackrel{\bmod C^{\infty}}{=} \sum_{j} U^{(j)}(t),
$$

where the phase function of each oscillatory integral $U^{(j)}(t)$ is associated with the corresponding Hamiltonian $h^{(j)}(x, \xi)$. The symbol of the oscillatory integral $U^{(j)}(t)$ is a complex-valued $m \times m$ matrix-function $u^{(j)}(t ; y, \eta)$, where $y=\left(y^{1}, \ldots, y^{n}\right)$ is the position of the source of the wave (i.e. this is the same $y$ that appears in formula (1.1)) and $\eta=\left(\eta_{1}, \ldots, \eta_{n}\right)$ is the corresponding dual variable (covector at the point $y$ ). When $|\eta| \rightarrow+\infty$, the symbol admits an asymptotic expansion

$$
u^{(j)}(t ; y, \eta)=u_{0}^{(j)}(t ; y, \eta)+u_{-1}^{(j)}(t ; y, \eta)+\cdots
$$

into components positively homogeneous in $\eta$, with the subscript indicating degree of homogeneity.

The formula for the principal symbol of the oscillatory integral $U^{(j)}(t)$ is known, see [16] and [11], and reads as follows:

$$
\begin{aligned}
u_{0}^{(j)}(t ; y, \eta)=\left[v^{(j)}\left(x^{(j)}(t ; y, \eta), \xi^{(j)}(t ; y, \eta)\right)\right]\left[v^{(j)}(y, \eta)\right]^{*} \\
\quad \times \exp \left(-i \int_{0}^{t} q^{(j)}\left(x^{(j)}(\tau ; y, \eta), \xi^{(j)}(\tau ; y, \eta)\right) d \tau\right),
\end{aligned}
$$

where $v^{(j)}(z, \zeta)$ is the normalised eigenvector of the principal symbol $A_{1}(z, \zeta)$ corresponding to the eigenvalue (Hamiltonian) $h^{(j)}(z, \zeta),\left(x^{(j)}(t ; y, \eta), \xi^{(j)}(t ; y, \eta)\right)$ is the Hamiltonian trajectory originating from the point $(y, \eta)$, i.e. solution of the system of ordinary differential equations (the dot denotes differentiation in $t$ )

$$
\dot{x}^{(j)}=h_{\xi}^{(j)}\left(x^{(j)}, \xi^{(j)}\right), \quad \dot{\xi}^{(j)}=-h_{x}^{(j)}\left(x^{(j)}, \xi^{(j)}\right)
$$

subject to the initial condition $\left.\left(x^{(j)}, \xi^{(j)}\right)\right|_{t=0}=(y, \eta)$,

$$
q^{(j)}: T^{\prime} M \longrightarrow \mathbb{R}
$$


is the function

$$
q^{(j)} \stackrel{\text { def }}{=}\left[v^{(j)}\right]^{*} A_{\text {sub }} v^{(j)}-\frac{i}{2}\left\{\left[v^{(j)}\right]^{*}, A_{1}-h^{(j)}, v^{(j)}\right\}-i\left[v^{(j)}\right]^{*}\left\{v^{(j)}, h^{(j)}\right\}
$$

and

$$
A_{\mathrm{sub}}(z, \zeta) \stackrel{\text { def }}{=} A_{0}(z, \zeta)+\frac{i}{2}\left(A_{1}\right)_{z^{\alpha} \zeta_{\alpha}}(z, \zeta)
$$

is the subprincipal symbol of the operator $A$, with the subscripts $z^{\alpha}$ and $\zeta_{\alpha}$ indicating partial derivatives and the repeated index $\alpha$ indicating summation over $\alpha=1, \ldots, n$. Curly brackets in formula (1.14) denote the Poisson bracket on matrix-functions

$$
\{P, R\} \stackrel{\text { def }}{=} P_{z^{\alpha}} R_{\zeta_{\alpha}}-P_{\zeta_{\alpha}} R_{z^{\alpha}}
$$

and its further generalisation

$$
\{P, Q, R\} \stackrel{\text { def }}{=} P_{z^{\alpha}} Q R_{\zeta_{\alpha}}-P_{\zeta_{\alpha}} Q R_{z^{\alpha}}
$$

As the derivation of formula (1.12) was previously performed only in theses [16] and [11], we repeat it in Sections 2 and 3 of our paper. Our derivation differs slightly from that in [16] and [11].

Formula (1.12) is invariant under changes of local coordinates on the manifold $M$, i.e. elements of the $m \times m$ matrix-function $u_{0}^{(j)}(t ; y, \eta)$ are scalars on $\mathbb{R} \times T^{\prime} M$. Moreover, formula (1.12) is invariant under the transformation of the eigenvector of the principal symbol

$$
v^{(j)} \longmapsto e^{i \phi^{(j)}} v^{(j)},
$$

where

$$
\phi^{(j)}: T^{\prime} M \longrightarrow \mathbb{R}
$$

is an arbitrary smooth function. When some quantity is defined up to the action of a certain transformation, theoretical physicists refer to such a transformation as a gauge transformation. We follow this tradition. Note that our particular gauge transformation (1.18) and (1.19) is quite common in quantum mechanics: when $\phi^{(j)}$ is a function of the position variable $x$ only (i.e. when $\phi^{(j)}: M \rightarrow \mathbb{R}$ ) this gauge transformation is associated with electromagnetism.

Both Y. Safarov [16] and W. J. Nicoll [11] assumed that the operator $A$ is semibounded from below but this assumption is not essential and their formula (1.12) remains true in the more general case that we are dealing with.

However, knowing the principal symbol (1.12) of the oscillatory integral $U^{(j)}(t)$ is not enough if one wants to derive two-term asymptotics (1.6) and (1.7). One needs information about $u_{-1}^{(j)}(t ; y, \eta)$, the component of the symbol of the oscillatory integral $U^{(j)}(t)$ which is positively homogeneous in $\eta$ of degree -1 , see formula (1.11), but here the problem is that $u_{-1}^{(j)}(t ; y, \eta)$ is not a true invariant in the sense that it depends on the choice of phase function in the oscillatory integral. We overcome this difficulty 
by observing that $U^{(j)}(0)$ is a pseudodifferential operator, hence, it has a well-defined subprincipal symbol $\left[U^{(j)}(0)\right]_{\text {sub. }}$. We prove that

$$
\operatorname{tr}\left[U^{(j)}(0)\right]_{\mathrm{sub}}=-i\left\{\left[v^{(j)}\right]^{*}, v^{(j)}\right\}
$$

and subsequently show that information contained in formulae (1.12) and (1.20) is sufficient for the derivation of two-term asymptotics (1.6) and (1.7).

Note that the RHS of formula (1.20) is invariant under the gauge transformation (1.18) and (1.19).

Formula (1.20) plays a central role in our paper. Sections 2 and 3 provide auxiliary material needed for the proof of formula (1.20), whereas the actual proof of formula (1.20) is given in Section 4.

Let us elaborate briefly on the geometric meaning of the RHS of (1.20) (a more detailed exposition is presented in Section 5). The eigenvector of the principal symbol is defined up to a gauge transformation (1.18) and (1.19) so it is natural to introduce a $\mathrm{U}(1)$ connection on $T^{\prime} M$ as follows: when parallel transporting an eigenvector of the principal symbol along a curve in $T^{\prime} M$ we require that the derivative of the eigenvector along the curve be orthogonal to the eigenvector itself. This is equivalent to the introduction of an (intrinsic) electromagnetic field on $T^{\prime} M$, with the $2 n$-component real quantity

$$
i\left(\left[v^{(j)}\right]^{*} v_{x^{\alpha}}^{(j)},\left[v^{(j)}\right]^{*} v_{\xi_{\gamma}}^{(j)}\right)
$$

playing the role of the electromagnetic covector potential. Our quantity (1.21) is a 1 -form on $T^{\prime} M$, rather than on $M$ itself as is the case in "traditional" electromagnetism. The above $\mathrm{U}(1)$ connection generates curvature which is a 2 -form on $T^{\prime} M$, an analogue of the electromagnetic tensor. Out of this curvature 2-form one can construct, by contraction of indices, a real scalar. This scalar curvature is the expression appearing in the RHS of formula (1.20).

Observe now that $\sum_{j} U^{(j)}(0)$ is the identity operator on half-densities. The subprincipal symbol of the identity operator is zero, so formula (1.20) implies

$$
\sum_{j}\left\{\left[v^{(j)}\right]^{*}, v^{(j)}\right\}=0
$$

One can check the identity (1.22) directly, without constructing the oscillatory integrals $U^{(j)}(t)$ : it follows from the fact that the $v^{(j)}(x, \xi)$ form an orthonormal basis, see end of Section 5 for details. We mentioned the identity (1.22) in order to highlight, once again, the fact that the curvature effects we have identified are specific to systems and do not have an analogue in the scalar case. 
Results 2 and 3. We prove, under appropriate assumptions on Hamiltonian trajectories (see Theorems 8.3 and 8.4), asymptotic formulae (1.6) and (1.7) with

$$
a(x)=\sum_{j=1}^{m^{+}} \int_{h^{(j)}(x, \xi)<1} d \xi,
$$

and

$$
\begin{aligned}
b(x)=-n \sum_{j=1}^{m^{+}} \int_{h^{(j)}(x, \xi)<1}\left(\left[v^{(j)}\right]^{*} A_{\mathrm{sub}} v^{(j)}\right. & -\frac{i}{2}\left\{\left[v^{(j)}\right]^{*}, A_{1}-h^{(j)}, v^{(j)}\right\} \\
& \left.+\frac{i}{n-1} h^{(j)}\left\{\left[v^{(j)}\right]^{*}, v^{(j)}\right\}\right)(x, \xi) d \xi,
\end{aligned}
$$

and $a$ and $b$ expressed via the above densities (1.23) and (1.24) as (1.8) and (1.9). In (1.23) and (1.24) $d \xi$ is shorthand for

$$
d \xi \stackrel{\text { def }}{=}(2 \pi)^{-n} d \xi=(2 \pi)^{-n} d \xi_{1} \ldots d \xi_{n},
$$

and the Poisson bracket on matrix-functions $\{\cdot, \cdot\}$ and its further generalisation $\{\cdot, \cdot, \cdot\}$ are defined by formulae (1.16) and (1.17) respectively.

To our knowledge, formula (1.24) is a new result. Note that in [16] this formula (more precisely, its integrated over $M$ version (1.9)) was written incorrectly, without the curvature terms $-\frac{n i}{n-1} \int h^{(j)}\left\{\left[v^{(j)}\right]^{*}, v^{(j)}\right\}$. See also Section 11 where we give a more detailed bibliographic review.

It is easy to see that the right-hand sides of (1.23) and (1.24) behave as densities under changes of local coordinates on the manifold $M$ and that these expressions are invariant under gauge transformations (1.18) and (1.19) of the eigenvectors of the principal symbol. Moreover, the right-hand sides of (1.23) and (1.24) are unitarily invariant, i.e. invariant under the transformation of the operator

$$
A \longmapsto R A R^{*},
$$

where

$$
R: M \longrightarrow \mathrm{U}(m)
$$

is an arbitrary smooth unitary matrix-function. The fact that the RHS of (1.24) is unitarily invariant is non-trivial: the appropriate calculations are presented in Section 9. The observation that without the curvature terms $-\frac{n i}{n-1} \int h^{(j)}\left\{\left[v^{(j)}\right]^{*}, v^{(j)}\right\}$ (as in [16]) the RHS of (1.24) is not unitarily invariant was a major motivating factor in the writing of this paper. 
Formula (1.24) is the main result of our paper. Note that even though the twoterm asymptotic expansion (1.6) holds only under certain assumptions on Hamiltonian trajectories (loops), the second asymptotic coefficient (1.24) is, in itself, well-defined irrespective of how many loops we have. If one wishes to reformulate the asymptotic expansion (1.6) in such a way that it remains valid without assumptions on the number of loops, this can easily be achieved, say, by taking a convolution with a function from Schwartz space $\delta(\mathbb{R})$ : see Theorem 7.1.

\section{Algorithm for the construction of the propagator}

We construct the propagator as a sum of $m$ oscillatory integrals (1.10) where each integral is of the form

$$
U^{(j)}(t)=\int e^{i \varphi^{(j)}(t, x ; y, \eta)} u^{(j)}(t ; y, \eta) \varsigma^{(j)}(t, x ; y, \eta) d_{\varphi^{(j)}}(t, x ; y, \eta)(\cdot) d y d \eta .
$$

Here we use notation from the book [18], only adapted to systems. Namely, the expressions appearing in formula (2.1) have the following meaning.

- The function $\varphi^{(j)}$ is a phase function, i.e. a function

$$
\varphi^{(j)}: \mathbb{R} \times M \times T^{\prime} M \longrightarrow \mathbb{C}
$$

positively homogeneous in $\eta$ of degree 1 and satisfying the conditions

$$
\begin{gathered}
\varphi^{(j)}(t, x ; y, \eta)=\left(x-x^{(j)}(t ; y, \eta)\right)^{\alpha} \xi_{\alpha}^{(j)}(t ; y, \eta) \\
+O\left(\left|x-x^{(j)}(t ; y, \eta)\right|^{2}\right), \\
\operatorname{Im} \varphi^{(j)}(t, x ; y, \eta) \geq 0,
\end{gathered}
$$

and

$$
\operatorname{det} \varphi_{x^{\alpha} \eta_{\beta}}^{(j)}\left(t, x^{(j)}(t ; y, \eta) ; y, \eta\right) \neq 0 .
$$

Recall that, according to Corollary 2.4.5 from [18], we are guaranteed to have inequality (2.4) if we choose a phase function

$$
\begin{aligned}
\varphi^{(j)}(t, x ; y, \eta)=( & \left.-x^{(j)}(t ; y, \eta)\right)^{\alpha} \xi_{\alpha}^{(j)}(t ; y, \eta) \\
& +\frac{1}{2} C_{\alpha \beta}^{(j)}(t ; y, \eta)\left(x-x^{(j)}(t ; y, \eta)\right)^{\alpha}\left(x-x^{(j)}(t ; y, \eta)\right)^{\beta} \\
& +O\left(\left|x-x^{(j)}(t ; y, \eta)\right|^{3}\right)
\end{aligned}
$$

with complex-valued symmetric matrix-function $C_{\alpha \beta}^{(j)}$ satisfying the strict inequality $\operatorname{Im} C^{(j)}>0$ (our original requirement (2.3) implies only the non-strict 
inequality $\operatorname{Im} C^{(j)} \geq 0$ ). Note that even though the matrix-function $C_{\alpha \beta}^{(j)}$ is not a tensor, the inequalities $\operatorname{Im} C^{(j)} \geq 0$ and $\operatorname{Im} C^{(j)}>0$ are invariant under transformations of local coordinates $x$; see Remark 2.4.9 in [18] for details.

- The quantity $u^{(j)}$ is the symbol of our oscillatory integral, i.e. a complex-valued $m \times m$ matrix-function $\mathbb{R} \times T^{\prime} M \rightarrow \mathbb{C}^{m^{2}}$ which admits the asymptotic expansion (1.11). The symbol is the unknown quantity in our construction.

- The quantity $d_{\varphi^{(j)}}$ is defined in accordance with formula (2.2.4) from [18] as

$$
d_{\varphi^{(j)}}(t, x ; y, \eta) \stackrel{\operatorname{def}}{=}\left(\operatorname{det}^{2} \varphi_{x^{\alpha} \eta_{\beta}}^{(j)}\right)^{1 / 4}=\left|\operatorname{det} \varphi_{x^{\alpha} \eta_{\beta}}^{(j)}\right|^{1 / 2} e^{i \arg \left(\operatorname{det}^{2} \varphi_{x^{\alpha} \eta_{\beta}}^{(j)}\right) / 4}
$$

Note that, in view of (2.4), our $d_{\varphi^{(j)}}$ is well-defined and smooth for $x$ close to $x^{(j)}(t ; y, \eta)$. It is known [18] that under coordinate transformations $d_{\varphi^{(j)}}$ behaves as a half-density in $x$ and as a half-density to the power -1 in $y$.

In formula (2.6) we wrote $\left(\operatorname{det}^{2} \varphi_{x^{\alpha} \eta_{\beta}}^{(j)}\right)^{1 / 4}$ rather than $\left(\operatorname{det} \varphi_{x^{\alpha} \eta_{\beta}}^{(j)}\right)^{1 / 2}$ in order to make this expression truly invariant under coordinate transformations. Recall that local coordinates $x$ and $y$ are chosen independently and that $\eta$ is a covector based at the point $y$. Consequently, $\operatorname{det} \varphi_{x^{\alpha} \eta_{\beta}}^{(j)}$ changes sign under inversion of one of the local coordinates $x^{\alpha}, \alpha=1, \ldots, n$, or $y^{\beta}, \beta=1, \ldots, n$, whereas $\operatorname{det}^{2} \varphi_{x^{\alpha} \eta_{\beta}}^{(j)}$ retains sign under inversion.

The choice of (smooth) branch of $\arg \left(\operatorname{det}^{2} \varphi_{x^{\alpha} \eta_{\beta}}^{(j)}\right)$ is assumed to be fixed. Thus, for a given phase function $\varphi^{(j)}$ formula (2.6) defines the quantity $d_{\varphi^{(j)}}$ uniquely up to a factor $e^{i k \pi / 2}, k=0,1,2,3$. Observe now that if we set $t=0$ and choose the same local coordinates for $x$ and $y$, we get $\varphi_{x^{\alpha} \eta_{\beta}}^{(j)}(0, y ; y, \eta)=I$. This implies that we can fully specify the choice of branch of $\arg \left(\operatorname{det}^{2} \varphi_{x^{\alpha} \eta_{\beta}}^{(j)}\right)$ by requiring that $d_{\varphi^{(j)}}(0, y ; y, \eta)=1$.

The purpose of the introduction of the factor $d_{\varphi^{(j)}}$ in (2.1) is twofold.

(a) It ensures that the symbol $u^{(j)}$ is a function on $\mathbb{R} \times T^{\prime} M$ in the full differential geometric sense of the word, i.e. that it is invariant under transformations of local coordinates $x$ and $y$.

(b) It ensures that the principal symbol $u_{0}^{(j)}$ does not depend on the choice of phase function $\varphi^{(j)}$. See Remark 2.2.8 in [18] for more details.

- The quantity $\varsigma^{(j)}$ is a smooth cut-off function $\mathbb{R} \times M \times T^{\prime} M \rightarrow \mathbb{R}$ satisfying the following conditions. 
(a) $\varsigma^{(j)}(t, x ; y, \eta)=0$ on the set $\left\{(t, x ; y, \eta):\left|h^{(j)}(y, \eta)\right| \leq 1 / 2\right\}$.

(b) $\varsigma^{(j)}(t, x ; y, \eta)=1$ on the intersection of a small conic neighbourhood of the set

$$
\left\{(t, x ; y, \eta): x=x^{(j)}(t ; y, \eta)\right\}
$$

with the set $\left\{(t, x ; y, \eta):\left|h^{(j)}(y, \eta)\right| \geq 1\right\}$.

(c) $\varsigma^{(j)}(t, x ; y, \lambda \eta)=\varsigma^{(j)}(t, x ; y, \eta)$ for $\left|h^{(j)}(y, \eta)\right| \geq 1, \lambda \geq 1$.

- It is known (see Section 2.3 in [18] for details) that Hamiltonian trajectories generated by a Hamiltonian $h^{(j)}(x, \xi)$ positively homogeneous in $\xi$ of degree 1 satisfy the identity

$$
\left(x_{\eta}^{(j)}\right)^{\alpha \beta} \xi_{\alpha}^{(j)}=0,
$$

where $\left(x_{\eta}^{(j)}\right)^{\alpha \beta} \stackrel{\text { def }}{=} \partial\left(x^{(j)}\right)^{\alpha} / \partial \eta_{\beta}$. Formulae (2.2) and (2.8) imply

$$
\varphi_{\eta}^{(j)}\left(t, x^{(j)}(t ; y, \eta) ; y, \eta\right)=0 .
$$

This allows us to apply the stationary phase method in the neighbourhood of the set (2.7) and disregard what happens away from it.

Our task now is to construct the symbols $u^{(j)}(t ; y, \eta), j=1, \ldots, m$, so that our oscillatory integrals $U^{(j)}(t), j=1, \ldots, m$, satisfy the dynamic equations

$$
\left(D_{t}+A\left(x, D_{x}\right)\right) U^{(j)}(t) \stackrel{\bmod C^{\infty}}{=} 0
$$

and initial condition

$$
\sum_{j} U^{(j)}(0) \stackrel{\bmod C^{\infty}}{=} I,
$$

where $I$ is the identity operator on half-densities; compare with formulae (1.3) and (1.2) and (1.10). Note that the pseudodifferential operator $A$ in formula (2.10) acts on the oscillatory integral $U(t)$ in the variable $x$; say, if $A$ is a differential operator this means that in order to evaluate $A U^{(j)}(t)$ one has to perform the appropriate differentiations of the oscillatory integral (2.1) in the variable $x$. Following the conventions of Section 3.3 of [18], we emphasise the fact that the pseudodifferential operator $A$ in formula (2.10) acts on the oscillatory integral $U(t)$ in the variable $x$ by writing this pseudodifferential operator as $A\left(x, D_{x}\right)$, where

$$
D_{x^{\alpha}} \stackrel{\text { def }}{=}-i \frac{\partial}{\partial x^{\alpha}} .
$$

We examine first the dynamic equation (2.10). We have

$$
\left(D_{t}+A\left(x, D_{x}\right)\right) U^{(j)}(t)=F^{(j)}(t),
$$


where $F^{(j)}(t)$ is the oscillatory integral

$$
F^{(j)}(t)=\int e^{i \varphi^{(j)}(t, x ; y, \eta)} f^{(j)}(t, x ; y, \eta) \varsigma^{(j)}(t, x ; y, \eta) d_{\varphi^{(j)}}(t, x ; y, \eta)(\cdot) d y d \eta
$$

whose matrix-valued amplitude $f^{(j)}$ is given by the formula

$$
f^{(j)}=D_{t} u^{(j)}+\left(\varphi_{t}^{(j)}+\left(d_{\varphi^{(j)}}\right)^{-1}\left(D_{t} d_{\varphi^{(j)}}\right)+s^{(j)}\right) u^{(j)},
$$

where the matrix-function $s^{(j)}(t, x ; y, \eta)$ is defined as

$$
s^{(j)}=e^{-i \varphi^{(j)}}\left(d_{\varphi^{(j)}}\right)^{-1} A\left(x, D_{x}\right)\left(e^{i \varphi^{(j)}} d_{\varphi^{(j)}}\right) .
$$

Theorem 18.1 from [19] gives us the following explicit asymptotic (in inverse powers of $\eta$ ) formula for the matrix-function (2.13):

$$
s^{(j)}=\left.\left(d_{\varphi^{(j)}}\right)^{-1} \sum_{\boldsymbol{\alpha}} \frac{1}{\boldsymbol{\alpha} !} A^{(\boldsymbol{\alpha})}\left(x, \varphi_{x}^{(j)}\right)\left(D_{z}^{\boldsymbol{\alpha}} \chi^{(j)}\right)\right|_{z=x}
$$

where

$$
\chi^{(j)}(t, z, x ; y, \eta)=e^{i \psi^{(j)}(t, z, x ; y, \eta)} d_{\varphi^{(j)}}(t, z ; y, \eta)
$$

and

$$
\psi^{(j)}(t, z, x ; y, \eta)=\varphi^{(j)}(t, z ; y, \eta)-\varphi^{(j)}(t, x ; y, \eta)-\varphi_{x^{\beta}}^{(j)}(t, x ; y, \eta)(z-x)^{\beta} .
$$

In formula (2.14)

- $\boldsymbol{\alpha} \stackrel{\text { def }}{=}\left(\alpha_{1}, \ldots, \alpha_{n}\right)$ is a multi-index (note the bold font which we use to distinguish multi-indices and individual indices),

$$
\begin{gathered}
\alpha ! \stackrel{\text { def }}{=} \alpha_{1} ! \ldots \alpha_{n} !, \\
D_{z}^{\alpha} \stackrel{\text { def }}{=} D_{z^{1}}^{\alpha_{1}} \ldots D_{z^{n}}^{\alpha_{n}},
\end{gathered}
$$

and

$$
D_{z} \stackrel{\text { def }}{=}-i \frac{\partial}{\partial z^{\beta}}
$$

- $A(x, \xi)$ is the full symbol of the pseudodifferential operator $A$ written in local coordinates $x$;

- we set

and

$$
\begin{array}{r}
A^{(\boldsymbol{\alpha})}(x, \xi) \stackrel{\text { def }}{=} \partial_{\xi}^{\boldsymbol{\alpha}} A(x, \xi), \\
\partial_{\xi}^{\boldsymbol{\alpha}} \stackrel{\text { def }}{=} \partial_{\xi_{1}}^{\alpha_{1}} \ldots \partial_{\xi_{n}}^{\alpha_{n}},
\end{array}
$$

$$
\partial_{\xi_{\beta}} \stackrel{\text { def }}{=} \frac{\partial}{\partial \xi_{\beta}}
$$


When $|\eta| \rightarrow+\infty$ the matrix-valued amplitude $f^{(j)}(t, x ; y, \eta)$ defined by formula (2.12) admits an asymptotic expansion

$$
f^{(j)}(t, x ; y, \eta)=f_{1}^{(j)}(t, x ; y, \eta)+f_{0}^{(j)}(t, x ; y, \eta)+f_{-1}^{(j)}(t, x ; y, \eta)+\cdots
$$

into components positively homogeneous in $\eta$, with the subscript indicating degree of homogeneity. Note the following differences between formulae (1.11) and (2.17).

- The leading term in (2.17) has degree of homogeneity 1 , rather than 0 as in (1.11). In fact, the leading term in (2.17) can be easily written out explicitly

$$
f_{1}^{(j)}(t, x ; y, \eta)=\left(\varphi_{t}^{(j)}(t, x ; y, \eta)+A_{1}\left(x, \varphi_{x}^{(j)}(t, x ; y, \eta)\right)\right) u_{0}^{(j)}(t ; y, \eta),
$$

where $A_{1}(x, \xi)$ is the (matrix-valued) principal symbol of the pseudodifferential operator $A$.

- Unlike the symbol $u^{(j)}(t ; y, \eta)$, the amplitude $f^{(j)}(t, x ; y, \eta)$ depends on $x$.

We now need to exclude the dependence on $x$ from the amplitude $f^{(j)}(t, x ; y, \eta)$. This can be done by means of the algorithm described in subsection 2.7.3 of [18]. We outline this algorithm below.

Working in local coordinates, define the matrix-function $\varphi_{x \eta}^{(j)}$ in accordance with

$$
\left(\varphi_{x \eta}^{(j)}\right)_{\alpha}^{\beta} \stackrel{\text { def }}{=} \varphi_{x^{\alpha} \eta_{\beta}}^{(j)}
$$

and then define its inverse $\left(\varphi_{x \eta}^{(j)}\right)^{-1}$ from the identity

$$
\left(\varphi^{(j)}\right)_{\alpha}^{\beta}\left[\left(\varphi_{x \eta}^{(j)}\right)^{-1}\right]_{\beta}^{\gamma}=\delta_{\alpha}^{\gamma}
$$

Define the "scalar" first order linear differential operators

$$
L_{\alpha}^{(j)} \stackrel{\text { def }}{=}\left[\left(\varphi_{x \eta}^{(j)}\right)^{-1}\right]_{\alpha}^{\beta}\left(\partial / \partial x^{\beta}\right), \quad \alpha=1, \ldots, n .
$$

Note that the coefficients of these differential operators are functions of the position variable $x$ and the dual variable $\xi$. It is known, see the second part of Appendix $\mathrm{E}$ in [18], that the operators (2.19) commute: $L_{\alpha}^{(j)} L_{\beta}^{(j)}=L_{\beta}^{(j)} L_{\alpha}^{(j)}, \alpha, \beta=1, \ldots, n$.

Denote

$$
\begin{gathered}
L_{\alpha}^{(j)} \stackrel{\text { def }}{=}\left(L_{1}^{(j)}\right)^{\alpha_{1}} \ldots\left(L_{n}^{(j)}\right)^{\alpha_{n}}, \\
\left(-\varphi_{\eta}^{(j)}\right)^{\alpha} \stackrel{\text { def }}{=}\left(-\varphi_{\eta_{1}}^{(j)}\right)^{\alpha_{1}} \ldots\left(-\varphi_{\eta_{n}}^{(j)}\right)^{\alpha_{n}},
\end{gathered}
$$

and, given an $r \in \mathbb{N}$, define the "scalar" linear differential operator

$$
\mathfrak{P}_{-1, r}^{(j)} \stackrel{\text { def }}{=} i\left(d_{\varphi^{(j)}}\right)^{-1} \frac{\partial}{\partial \eta_{\beta}} d_{\varphi^{(j)}}\left(1+\sum_{1 \leq|\boldsymbol{\alpha}| \leq 2 r-1} \frac{\left(-\varphi_{\eta}^{(j)}\right)^{\boldsymbol{\alpha}}}{\boldsymbol{\alpha} !(|\boldsymbol{\alpha}|+1)} L_{\boldsymbol{\alpha}}^{(j)}\right) L_{\beta}^{(j)},
$$


where $|\boldsymbol{\alpha}| \stackrel{\text { def }}{=} \alpha_{1}+\ldots+\alpha_{n}$ and the repeated index $\beta$ indicates summation over $\beta=1, \ldots, n$.

Recall Definition 2.7.8 from [18]: the linear operator $L$ is said to be positively homogeneous in $\eta$ of degree $p \in \mathbb{R}$ if for any $q \in \mathbb{R}$ and any function $f$ positively homogeneous in $\eta$ of degree $q$ the function $L f$ is positively homogeneous in $\eta$ of degree $p+q$. It is easy to see that the operator (2.20) is positively homogeneous in $\eta$ of degree -1 and the first subscript in $\mathfrak{P}_{-1, r}^{(j)}$ emphasises this fact.

Let $\mathfrak{S}_{0}^{(j)}$ be the (linear) operator of restriction to $x=x^{(j)}(t ; y, \eta)$,

$$
\left.\mathfrak{S}_{0}^{(j)} \stackrel{\text { def }}{=}(\cdot)\right|_{x=x^{(j)}(t ; y, \eta)},
$$

and let

$$
\mathfrak{S}_{-r}^{(j)} \stackrel{\text { def }}{=} \mathfrak{S}_{0}^{(j)}\left(\mathfrak{P}_{-1, r}^{(j)}\right)^{r}
$$

for $r=1,2, \ldots$ Observe that our linear operators $\mathfrak{S}_{-r}^{(j)}, r=0,1,2, \ldots$, are positively homogeneous in $\eta$ of degree $-r$. This observation allows us to define the linear operator

$$
\mathfrak{S}^{(j)} \stackrel{\text { def }}{=} \sum_{r=0}^{+\infty} \mathfrak{S}_{-r}^{(j)},
$$

where the series is understood as an asymptotic series in inverse powers of $\eta$.

According to subsection 2.7.3 of [18], the dynamic equation (2.10) can now be rewritten in the equivalent form

$$
\mathfrak{S}^{(j)} f^{(j)}=0,
$$

where the equality is understood in the asymptotic sense, as an asymptotic expansion in inverse powers of $\eta$. Recall that the matrix-valued amplitude $f^{(j)}(t, x ; y, \eta)$ appearing in (2.24) is defined by formulae (2.12)-(2.16).

Substituting (2.23) and (2.17) into (2.24) we obtain a hierarchy of equations

$$
\begin{gathered}
\mathfrak{S}_{0}^{(j)} f_{1}^{(j)}=0, \\
\mathfrak{S}_{-1}^{(j)} f_{1}^{(j)}+\mathfrak{S}_{0}^{(j)} f_{0}^{(j)}=0, \\
\mathfrak{S}_{-2}^{(j)} f_{1}^{(j)}+\mathfrak{S}_{-1}^{(j)} f_{0}^{(j)}+\mathfrak{S}_{0}^{(j)} f_{-1}^{(j)}=0,
\end{gathered}
$$

positively homogeneous in $\eta$ of degree $1,0,-1, \ldots$. These are the transport equations for the determination of the unknown homogeneous components $u_{0}^{(j)}(t ; y, \eta)$, $u_{-1}^{(j)}(t ; y, \eta), u_{-2}^{(j)}(t ; y, \eta), \ldots$, of the symbol of the oscillatory integral $(2.1)$.

Let us now examine the initial condition (2.11). Each operator $U^{(j)}(0)$ is a pseudodifferential operator, only written in a slightly nonstandard form. The issues here are as follows. 
- We use the invariantly defined phase function $\varphi^{(j)}(0, x ; y, \eta)=(x-y)^{\alpha} \eta_{\alpha}+$ $O\left(|x-y|^{2}\right)$ rather than the linear phase function $(x-y)^{\alpha} \eta_{\alpha}$ written in local coordinates.

- When defining the (full) symbol of the operator $U^{(j)}(t)$ we excluded the variable $x$ from the amplitude rather than the variable $y$. Note that when dealing with pseudodifferential operators it is customary to exclude the variable $y$ from the amplitude; exclusion of the variable $x$ gives the dual symbol of a pseudodifferential operator, see subsection 2.1.3 in [18]. Thus, at $t=0$, our symbol $u^{(j)}(0 ; y, \eta)$ resembles the dual symbol of a pseudodifferential operator rather than the "normal" symbol.

- We have the extra factor $d_{\varphi^{(j)}}(0, x ; y, \eta)$ in our representation of the operator $U^{(j)}(0)$ as an oscillatory integral.

The (full) dual symbol of the pseudodifferential operator $U^{(j)}(0)$ can be calculated in local coordinates in accordance with the following formula which addresses the issues highlighted above:

$$
\left.\sum_{\boldsymbol{\alpha}} \frac{(-1)^{|\boldsymbol{\alpha}|}}{\boldsymbol{\alpha} !}\left(D_{x}^{\boldsymbol{\alpha}} \partial_{\eta}^{\boldsymbol{\alpha}} u^{(j)}(0 ; y, \eta) e^{i \omega^{(j)}(x ; y, \eta)} d_{\varphi^{(j)}}(0, x ; y, \eta)\right)\right|_{x=y},
$$

where $\omega^{(j)}(x ; y, \eta)=\varphi^{(j)}(0, x ; y, \eta)-(x-y)^{\beta} \eta_{\beta}$. Formula (2.27) is a version of the formula from Section 2.1.3 of [18], only with the extra factor $(-1)^{|\alpha|}$. The latter is needed because we are writing down the dual symbol of the pseudodifferential operator $U^{(j)}(0)$ (no dependence on $x$ ) rather than its "normal" symbol (no dependence on $y$ ).

The initial condition (2.11) can now be rewritten in explicit form as

$$
\left.\sum_{j} \sum_{\boldsymbol{\alpha}} \frac{(-1)^{|\boldsymbol{\alpha}|}}{\boldsymbol{\alpha} !}\left(D_{x}^{\boldsymbol{\alpha}} \partial_{\eta}^{\boldsymbol{\alpha}} u^{(j)}(0 ; y, \eta) e^{i \omega^{(j)}(x ; y, \eta)} d_{\varphi^{(j)}}(0, x ; y, \eta)\right)\right|_{x=y}=I,
$$

where $I$ is the $m \times m$ identity matrix. Condition (2.28) can be decomposed into components positively homogeneous in $\eta$ of degree $0,-1,-2, \ldots$, giving us a hierarchy of initial conditions. The leading (of degree of homogeneity 0 ) initial condition reads

$$
\sum_{j} u_{0}^{(j)}(0 ; y, \eta)=I
$$

whereas lower order initial conditions are more complicated and depend on the choice of our phase functions $\varphi^{(j)}$. 


\section{Leading transport equations}

Formulae (2.21), (2.18), (2.2), (1.13) and the identity $\xi_{\alpha} h_{\xi_{\alpha}}^{(j)}(x, \xi)=h^{(j)}(x, \xi)$ (consequence of the fact that $h^{(j)}(x, \xi)$ is positively homogeneous in $\xi$ of degree 1) give us the following explicit representation for the leading transport equation (2.25):

$$
\left[A_{1}\left(x^{(j)}(t ; y, \eta), \xi^{(j)}(t ; y, \eta)\right)-h^{(j)}\left(x^{(j)}(t ; y, \eta), \xi^{(j)}(t ; y, \eta)\right)\right] u_{0}^{(j)}(t ; y, \eta)=0 .
$$

Here, of course, $h^{(j)}\left(x^{(j)}(t ; y, \eta), \xi^{(j)}(t ; y, \eta)\right)=h^{(j)}(y, \eta)$.

Equation (3.1) implies that

$$
u_{0}^{(j)}(t ; y, \eta)=v^{(j)}\left(x^{(j)}(t ; y, \eta), \xi^{(j)}(t ; y, \eta)\right)\left[w^{(j)}(t ; y, \eta)\right]^{T},
$$

where $v^{(j)}(z, \zeta)$ is the normalised eigenvector of the principal symbol $A_{1}(z, \zeta)$ corresponding to the eigenvalue $h^{(j)}(z, \zeta)$ and $w^{(j)}: \mathbb{R} \times T^{\prime} M \rightarrow \mathbb{C}^{m}$ is a columnfunction, positively homogeneous in $\eta$ of degree 0 , that remains to be found. Formulae (2.29) and (3.2) imply the following initial condition for the unknown columnfunction $w^{(j)}$ :

$$
w^{(j)}(0 ; y, \eta)=\overline{v^{(j)}(y, \eta)}
$$

We now consider the next transport equation in our hierarchy, equation (2.26). We will write down the two terms appearing in (2.26) separately.

In view of formulae (2.18) and (2.20)-(2.22), the first term in (2.26) reads

$$
\begin{aligned}
& \mathfrak{S}_{-1}^{(j)} f_{1}^{(j)} \\
& =\left.i\left[\left(d_{\varphi^{(j)}}\right)^{-1} \frac{\partial}{\partial \eta_{\beta}} d_{\varphi^{(j)}}\left(1-\frac{1}{2} \varphi_{\eta_{\alpha}}^{(j)} L_{\alpha}^{(j)}\right)\left(L_{\beta}^{(j)}\left(\varphi_{t}^{(j)}+A_{1}\left(x, \varphi_{x}^{(j)}\right)\right)\right) u_{0}^{(j)}\right]\right|_{x_{x=x^{(j)}}},
\end{aligned}
$$

where we dropped, for the sake of brevity, the arguments $(t ; y, \eta)$ in $u_{0}^{(j)}$ and $x^{(j)}$, and the arguments $(t, x ; y, \eta)$ in $\varphi_{t}^{(j)}, \varphi_{x}^{(j)}, \varphi_{\eta}^{(j)}$ and $d_{\varphi^{(j)}}$. Recall that the differential operators $L_{\alpha}^{(j)}$ are defined in accordance with formula (2.19) and the coefficients of these operators depend on $(t, x ; y, \eta)$.

In view of formulae (2.12)-(2.17) and (2.21), the second term in (2.26) reads

$$
\begin{aligned}
& \mathfrak{S}_{0}^{(j)} f_{0}^{(j)} \\
& =D_{t} u_{0}^{(j)} \\
& \quad+\left.\left[\left(d_{\varphi^{(j)}}\right)^{-1}\left(D_{t}+\left(A_{1}\right)_{\xi_{\alpha}} D_{x^{\alpha}}\right) d_{\varphi^{(j)}}+A_{0}-\frac{i}{2}\left(A_{1}\right)_{\xi_{\alpha} \xi_{\beta}} C_{\alpha \beta}^{(j)}\right]\right|_{x=x^{(j)}} u_{0}^{(j)} \\
& \quad+\left[A_{1}-h^{(j)}\right] u_{-1}^{(j)},
\end{aligned}
$$

where

$$
\left.C_{\alpha \beta}^{(j) \stackrel{\text { def }}{=}} \varphi_{x^{\alpha} x^{\beta}}^{(j)}\right|_{x=x^{(j)}}
$$


is the matrix-function from (2.5). In formulae (3.5) and (3.6) we dropped, for the sake of brevity, the arguments $(t ; y, \eta)$ in $u_{0}^{(j)}, u_{-1}^{(j)}, C_{\alpha \beta}^{(j)}$ and $x^{(j)}$, the arguments $\left(x^{(j)}(t ; y, \eta), \xi^{(j)}(t ; y, \eta)\right)$ in $A_{0}, A_{1},\left(A_{1}\right)_{\xi_{\alpha}},\left(A_{1}\right)_{\xi_{\alpha} \xi_{\beta}}$ and $h^{(j)}$, and the arguments $(t, x ; y, \eta)$ in $d_{\varphi^{(j)}}$ and $\varphi_{x^{\alpha} x^{\beta}}^{(j)}$.

Looking at (3.4) and (3.5) we see that the transport equation (2.26) has a complicated structure. Hence, in this section we choose not to perform the analysis of the full equation (2.26) and analyse only one particular subequation of this equation. Namely, observe that equation (2.26) is equivalent to $m$ subequations

$$
\begin{aligned}
& {\left[v^{(j)}\right]^{*}\left[\mathfrak{S}_{-1}^{(j)} f_{1}^{(j)}+\mathfrak{S}_{0}^{(j)} f_{0}^{(j)}\right]=0,} \\
& {\left[v^{(l)}\right]^{*}\left[\mathfrak{S}_{-1}^{(j)} f_{1}^{(j)}+\mathfrak{S}_{0}^{(j)} f_{0}^{(j)}\right]=0, \quad l \neq j,}
\end{aligned}
$$

where we dropped, for the sake of brevity, the arguments $\left(x^{(j)}(t ; y, \eta), \xi^{(j)}(t ; y, \eta)\right)$ in $\left[v^{(j)}\right]^{*}$ and $\left[v^{(l)}\right]^{*}$. In the remainder of this section we analyse (sub)equation (3.7) only.

Equation (3.7) is simpler than each of the $m-1$ equations (3.8) for the following two reasons.

- Firstly, the term $\left[A_{1}-h^{(j)}\right] u_{-1}^{(j)}$ from (3.5) vanishes after multiplication by $\left[v^{(j)}\right]^{*}$ from the left. Hence, equation (3.7) does not contain $u_{-1}^{(j)}$.

- Secondly, if we substitute (3.2) into (3.7), then the term with

$$
\partial\left[d_{\varphi^{(j)}} w^{(j)}(t ; y, \eta)\right]^{T} / \partial \eta_{\beta}
$$

vanishes. This follows from the fact that the scalar function

$$
\left[v^{(j)}\right]^{*}\left(\varphi_{t}^{(j)}+A_{1}\left(x, \varphi_{x}^{(j)}\right)\right) v^{(j)}
$$

has a second order zero, in the variable $x$, at $x=x^{(j)}(t ; y, \eta)$. Indeed, we have

$$
\begin{aligned}
& {\left.\left[\frac{\partial}{\partial x^{\alpha}}\left[v^{(j)}\right]^{*}\left(\varphi_{t}^{(j)}+A_{1}\left(x, \varphi_{x}^{(j)}\right)\right) v^{(j)}\right]\right|_{x=x^{(j)}}} \\
& \quad=\left.\left[v^{(j)}\right]^{*}\left[\left(\varphi_{t}^{(j)}+A_{1}\left(x, \varphi_{x}^{(j)}\right)\right)_{x^{\alpha}}\right]\right|_{x=x^{(j)}} v^{(j)} \\
& \quad=\left[v^{(j)}\right]^{*}\left(-h_{x^{\alpha}}^{(j)}-C_{\alpha \beta}^{(j)} h_{\xi_{\beta}}^{(j)}+\left(A_{1}\right)_{x^{\alpha}}+C_{\alpha \beta}^{(j)}\left(A_{1}\right)_{\xi_{\beta}}\right) v^{(j)} \\
& \quad=\left[v^{(j)}\right]^{*}\left(A_{1}\right)_{x^{\alpha}} v^{(j)}-h_{x^{\alpha}}^{(j)}+C_{\alpha \beta}^{(j)}\left(\left[v^{(j)}\right]^{*}\left(A_{1}\right)_{\xi_{\beta}} v^{(j)}-h_{\xi_{\beta}}^{(j)}\right) \\
& \quad=0,
\end{aligned}
$$

where in the last two lines we dropped, for the sake of brevity, the arguments $\left(x^{(j)}(t ; y, \eta), \xi^{(j)}(t ; y, \eta)\right)$ in $\left(A_{1}\right)_{x^{\alpha}},\left(A_{1}\right)_{\xi_{\beta}}, h_{x^{\alpha}}^{(j)}, h_{\xi_{\beta}}^{(j)}$, and the argument $(t ; y, \eta)$ in $C_{\alpha \beta}^{(j)}$ (the latter is the matrix-function from formulae (2.5) and (3.6)). Throughout the above argument we used the fact that our $\left[v^{(j)}\right]^{*}$ and $v^{(j)}$ do not depend on $x$ : their argument is $\left(x^{(j)}(t ; y, \eta), \xi^{(j)}(t ; y, \eta)\right)$. 
Substituting (3.4), (3.5), and (3.2) into (3.7) we get

$$
\left(D_{t}+p^{(j)}(t ; y, \eta)\right)\left[w^{(j)}(t ; y, \eta)\right]^{T}=0,
$$

where

$$
\begin{aligned}
p^{(j)}= & \left.i\left[v^{(j)}\right]^{*}\left[\frac{\partial}{\partial \eta_{\beta}}\left(1-\frac{1}{2} \varphi_{\eta_{\alpha}}^{(j)} L_{\alpha}^{(j)}\right)\left(L_{\beta}^{(j)}\left(\varphi_{t}^{(j)}+A_{1}\left(x, \varphi_{x}^{(j)}\right)\right)\right) v^{(j)}\right]\right|_{x_{x=x^{(j)}}} \\
& -i\left[v^{(j)}\right]^{*}\left\{v^{(j)}, h^{(j)}\right\}+\left.\left[\left(d_{\varphi^{(j)}}\right)^{-1}\left(D_{t}+h_{\xi_{\alpha}}^{(j)} D_{x^{\alpha}}\right) d_{\varphi^{(j)}}\right]\right|_{x_{x=x^{(j)}}} \\
& +\left[v^{(j)}\right]^{*}\left(A_{0}-\frac{i}{2}\left(A_{1}\right)_{\xi_{\alpha} \xi_{\beta}} C_{\alpha \beta}^{(j)}\right) v^{(j)} .
\end{aligned}
$$

Note that the ordinary differential operator in the LHS of formula (3.9) is a scalar one, i.e. it does not mix up the different components of the column-function $w^{(j)}(t ; y, \eta)$. The solution of the ordinary differential equation (3.9) subject to the initial condition (3.3) is

$$
w^{(j)}(t ; y, \eta)=\overline{v^{(j)}(y, \eta)} \exp \left(-i \int_{0}^{t} p^{(j)}(\tau ; y, \eta) d \tau\right) .
$$

Comparing formulae (3.2) and (3.11) with formula (1.12) we see that in order to prove the latter we need only to establish the scalar identity

$$
p^{(j)}(t ; y, \eta)=q^{(j)}\left(x^{(j)}(t ; y, \eta), \xi^{(j)}(t ; y, \eta)\right)
$$

where $q^{(j)}$ is the function (1.14). In view of the definitions of the quantities $p^{(j)}$ and $q^{(j)}$, see formulae (3.10) and (1.14), and the definition of the subprincipal symbol (1.15), proving the identity (3.12) reduces to proving the identity

$$
\begin{aligned}
\left\{\left[v^{(j)}\right]^{*},\right. & \left.A_{1}-h^{(j)}, v^{(j)}\right\}\left(x^{(j)}, \xi^{(j)}\right) \\
=-2\left[v^{(j)}\left(x^{(j)}, \xi^{(j)}\right)\right]^{*}\left[\frac{\partial}{\partial \eta_{\beta}}\left(1-\frac{1}{2} \varphi_{\eta_{\alpha}}^{(j)} L_{\alpha}^{(j)}\right)\right. & \left.\left(L_{\beta}^{(j)}\left(\varphi_{t}^{(j)}+A_{1}\left(x, \varphi_{x}^{(j)}\right)\right)\right) v^{(j)}\left(x^{(j)}, \xi^{(j)}\right)\right]\left.\right|_{x=x^{(j)}} \\
& +\left.2\left[\left(d_{\varphi^{(j)}}\right)^{-1}\left(\partial_{t}+h_{\xi_{\alpha}}^{(j)} \partial_{x^{\alpha}}\right) d_{\varphi^{(j)}}\right]\right|_{x=x^{(j)}} \\
& +\left[v^{(j)}\left(x^{(j)}, \xi^{(j)}\right)\right]^{*}\left(\left(A_{1}\right)_{x^{\alpha} \xi_{\alpha}}+\left(A_{1}\right) \xi_{\alpha} \xi_{\beta} C_{\alpha \beta}^{(j)}\right) v^{(j)}\left(x^{(j)}, \xi^{(j)}\right) .
\end{aligned}
$$

Note that the expressions in the LHS and RHS of (3.13) have different structure. The LHS of (3.13) is the generalised Poisson bracket $\left\{\left[v^{(j)}\right]^{*}, A_{1}-h^{(j)}, v^{(j)}\right\}$, see (1.17), evaluated at $z=x^{(j)}(t ; y, \eta), \zeta=\xi^{(j)}(t ; y, \eta)$, whereas the RHS of (3.13) involves partial derivatives (in $\eta$ ) of $v^{(j)}\left(x^{(j)}(t ; y, \eta), \xi^{(j)}(t ; y, \eta)\right)$ (Chain Rule). In writing (3.13) we also dropped, for the sake of brevity, the arguments $(t, x ; y, \eta)$ in $\varphi_{t}^{(j)}$, 
$\varphi_{x}^{(j)}, \varphi_{\eta}^{(j)}, d_{\varphi^{(j)}}$ and the coefficients of the differential operators $L_{\alpha}^{(j)}$ and $L_{\beta}^{(j)}$, the arguments $\left(x^{(j)}, \xi^{(j)}\right)$ in $h_{\xi_{\alpha}}^{(j)},\left(A_{1}\right)_{x^{\alpha} \xi_{\alpha}}$ and $\left(A_{1}\right)_{\xi_{\alpha} \xi_{\beta}}$, and the arguments $(t ; y, \eta)$ in $x^{(j)}, \xi^{(j)}$ and $C_{\alpha \beta}^{(j)}$.

Before performing the calculations that will establish the identity (3.13) we make several observations that will allow us to simplify these calculations considerably.

Firstly, our function $p^{(j)}(t ; y, \eta)$ does not depend on the choice of the phase function $\varphi^{(j)}(t, x ; y, \eta)$. Indeed, if $p^{(j)}(t ; y, \eta)$ did depend on the choice of phase function, then, in view of formulae (3.2) and (3.11) the principal symbol of our oscillatory integral $U^{(j)}(t)$ would depend on the choice of phase function, which would contradict Theorem 2.7.11 from [18]. Here we use the fact that operators $U^{(j)}(t)$ with different $j$ cannot compensate each other to give an integral operator whose integral kernel is infinitely smooth in $t, x$ and $y$ because all our $U^{(j)}(t)$ oscillate in $t$ in a different way: $\varphi_{t}^{(j)}\left(t, x^{(j)}(t ; y, \eta) ; y, \eta\right)=-h^{(j)}(y, \eta)$ and we assumed the eigenvalues $h^{(j)}(y, \eta)$ of our principal symbol $A_{1}(y, \eta)$ to be simple.

Secondly, the arguments (free variables) in (3.13) are $(t ; y, \eta)$. We fix an arbitrary point $(\tilde{t} ; \tilde{y}, \tilde{\eta}) \in \mathbb{R} \times T^{\prime} M$ and prove formula (3.13) at this point. Put

$$
\left(\xi_{\eta}^{(j)}\right)_{\alpha}^{\beta} \stackrel{\text { def }}{=} \partial\left(\xi^{(j)}\right)_{\alpha} / \partial \eta_{\beta}
$$

According to Lemma 2.3.2 from [18] there exists a local coordinate system $x$ such that $\operatorname{det}\left(\xi_{\eta}^{(j)}\right)_{\alpha}^{\beta} \neq 0$. This opens the way to the use of the linear phase function

$$
\varphi^{(j)}(t, x ; y, \eta)=\left(x-x^{(j)}(t ; y, \eta)\right)^{\alpha} \xi_{\alpha}^{(j)}(t ; y, \eta)
$$

which will simplify calculations to a great extent. Moreover, we can choose a local coordinate system $y$ such that

$$
\left(\xi_{\eta}^{(j)}\right)_{\alpha}^{\beta}(\tilde{t} ; \tilde{y}, \tilde{\eta})=\delta_{\alpha}{ }^{\beta}
$$

which will simplify calculations even further.

The calculations we are about to perform will make use of the symmetry

$$
\left(x_{\eta}^{(j)}\right)^{\gamma \alpha}\left(\xi_{\eta}^{(j)}\right) \gamma^{\beta}=\left(x_{\eta}^{(j)}\right)^{\gamma \beta}\left(\xi_{\eta}^{(j)}\right)_{\gamma}^{\alpha}
$$

which is an immediate consequence of formula (2.8). Formula (3.16) appears as formula (2.3.3) in [18] and the accompanying text explains its geometric meaning. Note that at the point $(\tilde{t} ; \tilde{y}, \tilde{\eta})$ formula (3.16) takes the especially simple form

$$
\left(x_{\eta}^{(j)}\right)^{\alpha \beta}(\tilde{t} ; \tilde{y}, \tilde{\eta})=\left(x_{\eta}^{(j)}\right)^{\beta \alpha}(\tilde{t} ; \tilde{y}, \tilde{\eta}) .
$$

Our calculations will also involve the quantity $\varphi_{\eta_{\alpha} \eta_{\beta}}^{(j)}(\tilde{t}, \tilde{x} ; \tilde{y}, \tilde{\eta})$ where

$$
\tilde{x} \stackrel{\text { def }}{=} x^{(j)}(\tilde{t} ; \tilde{y}, \tilde{\eta}) \text {. }
$$


Formulae (3.14), (2.8), (3.15), and (3.17) imply

$$
\varphi_{\eta_{\alpha} \eta_{\beta}}^{(j)}(\tilde{t}, \tilde{x} ; \tilde{y}, \tilde{\eta})=-\left(x_{\eta}^{(j)}\right)^{\alpha \beta}(\tilde{t} ; \tilde{y}, \tilde{\eta}) .
$$

Further on we denote

$$
\tilde{\xi} \stackrel{\text { def }}{=} \xi^{(j)}(\tilde{t} ; \tilde{y}, \tilde{\eta}) .
$$

With account of all the simplifications listed above, we can rewrite formula (3.13), which is the identity that we are proving, as

$$
\begin{aligned}
& \left\{\left[v^{(j)}\right]^{*}, A_{1}-h^{(j)}, v^{(j)}\right\}(\tilde{x}, \tilde{\xi}) \\
& =-2\left[\tilde{v}^{(j)}\right]^{*}\left[\frac { \partial ^ { 2 } } { \partial x ^ { \alpha } \partial \eta _ { \alpha } } \left(A_{1}\left(x, \xi^{(j)}\right)-h^{(j)}(\tilde{y}, \eta)\right.\right. \\
& \left.\left.\quad-\left(x-x^{(j)}\right)^{\gamma} h_{x^{\nu}}^{(j)}\left(x^{(j)}, \xi^{(j)}\right)\right) v^{(j)}\left(x^{(j)}, \xi^{(j)}\right)\right]\left.\right|_{(x, \eta)=(\tilde{x}, \tilde{\eta})} \\
& -\left(\tilde{x}_{\eta}^{(j)}\right)^{\alpha \beta}\left[\tilde{v}^{(j)}\right]^{*}\left[\frac { \partial ^ { 2 } } { \partial x ^ { \alpha } \partial x ^ { \beta } } \left(A_{1}\left(x, \xi^{(j)}\right)-h^{(j)}(\tilde{y}, \eta)\right.\right. \\
& \left.\left.\quad-\left(x-x^{(j)}\right)^{\gamma} h_{x^{\nu}}^{(j)}\left(x^{(j)}, \xi^{(j)}\right)\right) v^{(j)}\left(x^{(j)}, \xi^{(j)}\right)\right]\left.\right|_{(x, \eta)=(\tilde{x}, \tilde{\eta})} \\
& +\left[\tilde{v}^{(j)}\right]^{*}\left(\tilde{A_{1}}\right)_{x^{\alpha} \xi_{\alpha}} \tilde{v}^{(j)}-\tilde{h}_{x^{\alpha} \xi_{\alpha}}^{(j)}-\tilde{h}_{x^{\alpha} x^{\beta}}^{(j)}\left(\tilde{x}_{\eta}^{(j)}\right)^{\alpha \beta},
\end{aligned}
$$

where $\tilde{v}^{(j)}=v^{(j)}(\tilde{x}, \tilde{\xi}), \tilde{x}_{\eta}^{(j)}=x_{\eta}^{(j)}(\tilde{t} ; \tilde{y}, \tilde{\eta}),\left(\tilde{A}_{1}\right)_{x^{\alpha} \xi_{\alpha}}=\left(A_{1}\right)_{x^{\alpha} \xi_{\alpha}}(\tilde{x}, \tilde{\xi}), \tilde{h}_{x^{\alpha} \xi_{\alpha}}^{(j)}=$ $h_{x^{\alpha} \xi_{\alpha}}^{(j)}(\tilde{x}, \tilde{\xi}), \tilde{h}_{x^{\alpha} x^{\beta}}^{(j)}=h_{x^{\alpha} x^{\beta}}^{(j)}(\tilde{x}, \tilde{\xi}), x^{(j)}=x^{(j)}(\tilde{t} ; \tilde{y}, \eta)$, and $\xi^{(j)}=\xi^{(j)}(\tilde{t} ; \tilde{y}, \eta)$.

Note that the last two terms in the RHS of (3.19) originate from the term with $d_{\varphi^{(j)}}$ in (3.13): we used the fact that $d_{\varphi^{(j)}}$ does not depend on $x$ and that

$$
\left.\left[\left(d_{\varphi^{(j)}}\right)^{-1} \partial_{t} d_{\varphi^{(j)}}\right]\right|_{(t, x ; y, \eta)=(\tilde{t}, \tilde{x} ; \tilde{y}, \tilde{\eta})}=-\frac{1}{2}\left(\tilde{h}_{x^{\alpha} \xi_{\alpha}}^{(j)}+\tilde{h}_{x^{\alpha} x^{\beta}}^{(j)}\left(\tilde{x}_{\eta}^{(j)}\right)^{\alpha \beta}\right) .
$$

Formula (3.20) is a special case of formula (3.3.21) from [18].

Note also that the term $-h^{(j)}(\tilde{y}, \eta)$ appearing (twice) in the RHS of (3.19) will vanish after being acted upon with the differential operators $\frac{\partial^{2}}{\partial x^{\alpha} \partial \eta_{\alpha}}$ and $\frac{\partial^{2}}{\partial x^{\alpha} \partial x^{\beta}}$ because it does not depend on $x$.

We have

$$
\begin{aligned}
{\left[\tilde{v}^{(j)}\right]^{*} } & {\left[\frac { \partial ^ { 2 } } { \partial x ^ { \alpha } \partial \eta _ { \alpha } } \left(A_{1}\left(x, \xi^{(j)}\right)\right.\right.} \\
& \left.\left.\quad-\left(x-x^{(j)}\right)^{\gamma} h_{x^{\nu}}^{(j)}\left(x^{(j)}, \xi^{(j)}\right)\right) v^{(j)}\left(x^{(j)}, \xi^{(j)}\right)\right]\left.\right|_{(x, \eta)=(\tilde{x}, \tilde{\eta})} \\
= & {\left[\tilde{v}^{(j)}\right]^{*}\left(\tilde{A}_{1}\right)_{x^{\alpha} \xi_{\alpha}} \tilde{v}^{(j)}-\tilde{h}_{x^{\alpha} \xi_{\alpha}}^{(j)}-\tilde{h}_{x^{\alpha} x^{\beta}}^{(j)}\left(\tilde{x}_{\eta}^{(j)}\right)^{\alpha \beta} } \\
& +\left[\tilde{v}^{(j)}\right]^{*}\left(\left(\tilde{A}_{1}\right)_{x^{\alpha}}-\tilde{h}_{x^{\alpha}}^{(j)}\right)\left(\tilde{v}_{\xi_{\alpha}}^{(j)}+\tilde{v}_{x^{\beta}}^{(j)}\left(\tilde{x}_{\eta}^{(j)}\right)^{\alpha \beta}\right),
\end{aligned}
$$


and

$$
\begin{aligned}
& {\left[\tilde{v}^{(j)}\right]^{*}\left[\frac { \partial ^ { 2 } } { \partial x ^ { \alpha } \partial x ^ { \beta } } \left(A_{1}\left(x, \xi^{(j)}\right)\right.\right.} \\
& \left.\left.\quad-\left(x-x^{(j)}\right)^{\gamma} h_{x^{\nu}}^{(j)}\left(x^{(j)}, \xi^{(j)}\right)\right) v^{(j)}\left(x^{(j)}, \xi^{(j)}\right)\right]\left.\right|_{(x, \eta)=(\tilde{x}, \tilde{\eta})} \\
& \quad=\left[\tilde{v}^{(j)}\right]^{*}\left(\tilde{A}_{1}\right)_{x^{\alpha} x^{\beta}} \tilde{v}^{(j)},
\end{aligned}
$$

where $\left(\tilde{A}_{1}\right)_{x^{\alpha}}=\left(A_{1}\right)_{x^{\alpha}}(\tilde{x}, \tilde{\xi}), \tilde{h}_{x^{\alpha}}^{(j)}=h_{x^{\alpha}}^{(j)}(\tilde{x}, \tilde{\xi}), \tilde{v}_{\xi_{\alpha}}^{(j)}=v_{\xi_{\alpha}}^{(j)}(\tilde{x}, \tilde{\xi})$, and $\tilde{v}_{x^{\beta}}^{(j)}=$ $v_{x^{\beta}}^{(j)}(\tilde{x}, \tilde{\xi})$. We also have

$$
\begin{aligned}
& {\left[\tilde{v}^{(j)}\right]^{*}\left(\left(\tilde{A}_{1}\right)_{x^{\alpha}}-\tilde{h}_{x^{\alpha}}^{(j)}\right) \tilde{v}_{x^{\beta}}^{(j)}+\left[\tilde{v}^{(j)}\right]^{*}\left(\left(\tilde{A}_{1}\right)_{x^{\beta}}-\tilde{h}_{x^{\beta}}^{(j)}\right) \tilde{v}_{x^{\alpha}}^{(j)}} \\
& =\tilde{h}_{x^{\alpha} x^{\beta}}^{(j)}-\left[\tilde{v}^{(j)}\right]^{*}\left(\tilde{A}_{1}\right)_{x^{\alpha} x^{\beta}} \tilde{v}^{(j)} .
\end{aligned}
$$

Using formulae (3.23) and (3.17) we can rewrite formula (3.21) as

$$
\begin{aligned}
& {\left[\tilde{v}^{(j)}\right]^{*}\left[\frac { \partial ^ { 2 } } { \partial x ^ { \alpha } \partial \eta _ { \alpha } } \left(A_{1}\left(x, \xi^{(j)}\right)\right.\right.} \\
& \left.\left.\quad-\left(x-x^{(j)}\right)^{\gamma} h_{x^{\nu}}^{(j)}\left(x^{(j)}, \xi^{(j)}\right)\right) v^{(j)}\left(x^{(j)}, \xi^{(j)}\right)\right]\left.\right|_{(x, \eta)=(\tilde{x}, \tilde{\eta})} \\
& =\left[\tilde{v}^{(j)}\right]^{*}\left(\tilde{A}_{1}\right)_{x^{\alpha} \xi_{\alpha}} \tilde{v}^{(j)}-\tilde{h}_{x^{\alpha} \xi_{\alpha}}^{(j)}+\left[\tilde{v}^{(j)}\right]^{*}\left(\left(\tilde{A}_{1}\right)_{x^{\alpha}}-\tilde{h}_{x^{\alpha}}^{(j)}\right) \tilde{v}_{\xi_{\alpha}}^{(j)} \\
& \quad-\frac{1}{2}\left(\left[\tilde{v}^{(j)}\right]^{*}\left(\tilde{A}_{1}\right)_{x^{\alpha} x^{\beta}} \tilde{v}^{(j)}+\tilde{h}_{x^{\alpha} x^{\beta}}^{(j)}\left(\tilde{x}_{\eta}^{(j)}\right)^{\alpha \beta} .\right.
\end{aligned}
$$

Substituting (3.24) and (3.22) into (3.19) we see that all the terms with $\left(\tilde{x}_{\eta}^{(j)}\right)^{\alpha \beta}$ cancel out and we get

$$
\begin{aligned}
& \left\{\left[v^{(j)}\right]^{*}, A_{1}-h^{(j)}, v^{(j)}\right\}(\tilde{x}, \tilde{\xi}) \\
& \quad=-\left[\tilde{v}^{(j)}\right]^{*}\left(\left(\widetilde{A_{1}}\right)_{x^{\alpha} \xi_{\alpha}}-\tilde{h}_{x^{\alpha} \xi_{\alpha}}^{(j)}\right) \tilde{v}^{(j)}-2\left[\tilde{v}^{(j)}\right]^{*}\left(\left(\tilde{A}_{1}\right)_{x^{\alpha}}-\tilde{h}_{x^{\alpha}}^{(j)}\right) \tilde{v}_{\xi_{\alpha}}^{(j)} .
\end{aligned}
$$

Thus, the proof of the identity (3.13) has been reduced to the proof of the identity (3.25).

Observe now that formula (3.25) no longer has Hamiltonian trajectories present in it. This means that we can drop all the tildes and rewrite (3.25) as

$$
\begin{aligned}
& \left\{\left[v^{(j)}\right]^{*}, A_{1}-h^{(j)}, v^{(j)}\right\} \\
& \quad=-\left[v^{(j)}\right]^{*}\left(A_{1}-h^{(j)}\right)_{x^{\alpha} \xi_{\alpha}} v^{(j)}-2\left[v^{(j)}\right]^{*}\left(A_{1}-h^{(j)}\right)_{x} v_{\xi_{\alpha}}^{(j)},
\end{aligned}
$$

where the arguments are $(x, \xi)$. We no longer need to restrict our consideration to the particular point $(x, \xi)=(\tilde{x}, \tilde{\xi})$ : if we prove (3.26) for an arbitrary $(x, \xi) \in T^{\prime} M$ we will prove it for a particular $(\tilde{x}, \tilde{\xi}) \in T^{\prime} M$. 
The proof of the identity (3.26) is straightforward. We note that

$$
\begin{aligned}
& {\left[v^{(j)}\right]^{*}\left(A_{1}-h^{(j)}\right)_{x} \alpha \xi_{\alpha} v^{(j)}} \\
& \quad=-\left[v^{(j)}\right]^{*}\left(A_{1}-h^{(j)}\right)_{x} v_{\xi_{\alpha}}^{(j)}-\left[v^{(j)}\right]^{*}\left(A_{1}-h^{(j)}\right)_{\xi_{\alpha}} v_{x^{\alpha}}^{(j)}
\end{aligned}
$$

and substituting (3.27) into (3.26) reduce the latter to the form

$$
\begin{aligned}
& \left\{\left[v^{(j)}\right]^{*}, A_{1}-h^{(j)}, v^{(j)}\right\} \\
& \quad=\left[v^{(j)}\right]^{*}\left(A_{1}-h^{(j)}\right) \xi_{\alpha} v_{x^{\alpha}}^{(j)}-\left[v^{(j)}\right]^{*}\left(A_{1}-h^{(j)}\right)_{x} \alpha v_{\xi_{\alpha}}^{(j)} .
\end{aligned}
$$

But we have

$$
\left[v^{(j)}\right]^{*}\left(A_{1}-h^{(j)}\right)_{x^{\alpha}}=-\left[v_{x^{\alpha}}^{(j)}\right]^{*}\left(A_{1}-h^{(j)}\right),
$$

and

$$
\left[v^{(j)}\right]^{*}\left(A_{1}-h^{(j)}\right)_{\xi_{\alpha}}=-\left[v_{\xi_{\alpha}}^{(j)}\right]^{*}\left(A_{1}-h^{(j)}\right) .
$$

Substituting (3.29) and (3.30) into (3.28) we get

$$
\left\{\left[v^{(j)}\right]^{*}, A_{1}-h^{(j)}, v^{(j)}\right\}=\left[v_{x^{\alpha}}^{(j)}\right]^{*}\left(A_{1}-h^{(j)}\right) v_{\xi_{\alpha}}^{(j)}-\left[v_{\xi_{\alpha}}^{(j)}\right]^{*}\left(A_{1}-h^{(j)}\right) v_{x^{\alpha}}^{(j)},
$$

which agrees with the definition of the generalised Poisson bracket (1.17).

\section{Proof of formula (1.20)}

In this section we prove formula (1.20). Our approach is as follows.

We write down explicitly the transport equations (3.8) at $t=0$, i.e.

$$
\left.\left[v^{(l)}\right]^{*}\left[\mathfrak{S}_{-1}^{(j)} f_{1}^{(j)}+\mathfrak{S}_{0}^{(j)} f_{0}^{(j)}\right]\right|_{t=0}=0, \quad l \neq j .
$$

We use the same local coordinates for $x$ and $y$ and we assume all our phase functions to be linear, i.e. we assume that for each $j$ we have (3.14). Using linear phase functions is justified for small $t$ because we have $\left(\xi_{\eta}^{(j)}\right)_{\alpha}{ }^{\beta}(0 ; y, \eta)=\delta_{\alpha}{ }^{\beta}$ and, hence, $\operatorname{det} \varphi_{x^{\alpha} \eta_{\beta}}^{(j)}(t, x ; y, \eta) \neq 0$ for small $t$. Writing down equations (4.1) for linear phase functions is much easier than for general phase functions (2.2).

Using linear phase functions has the additional advantage that the initial condition (2.28) simplifies and reads now $\sum_{j} u^{(j)}(0 ; y, \eta)=I$. In view of (1.11), this implies, in particular, that

$$
\sum_{j} u_{-1}^{(j)}(0)=0 .
$$

Here and further on in this section we drop, for the sake of brevity, the arguments $(y, \eta)$ in $u_{-1}^{(j)}$. 
Of course, the formula we are proving, formula (1.20), does not depend on our choice of phase functions. It is just easier to carry out calculations for linear phase functions.

We will show that (4.1) is a system of complex linear algebraic equations for the unknowns $u_{-1}^{(j)}(0)$. The total number of equations (4.1) is $m^{2}-m$. However, for each $j$ and $l$ the LHS of (4.1) is a row of $m$ elements, so (4.1) is, effectively, a system of $m\left(m^{2}-m\right)$ scalar equations.

Equation (4.2) is a single matrix equation, so it is, effectively, a system of $m^{2}$ scalar equations.

Consequently, the system (4.1)-(4.2) is, effectively, a system of $m^{3}$ scalar equations. This is exactly the number of unknown scalar elements in the $m$ matrices $u_{-1}^{(j)}(0)$.

In the remainder of this section we write down explicitly the LHS of (4.1) and solve the linear algebraic system (4.1) and (4.2) for the unknowns $u_{-1}^{(j)}(0)$. This will allow us to prove formula (1.20).

Before starting explicit calculations we observe that equations (4.1) can be equivalently rewritten as

$$
\left.P^{(l)}\left[\mathfrak{S}_{-1}^{(j)} f_{1}^{(j)}+\mathfrak{S}_{0}^{(j)} f_{0}^{(j)}\right]\right|_{t=0}=0, \quad l \neq j,
$$

where

$$
P^{(l)} \stackrel{\text { def }}{=}\left[v^{(l)}(y, \eta)\right]\left[v^{(l)}(y, \eta)\right]^{*}
$$

is the orthogonal projection onto the eigenspace corresponding to the (normalised) eigenvector $v^{(l)}(y, \eta)$ of the principal symbol. We will deal with (4.3) rather than with (4.1). This is simply a matter of convenience.

4.1. Part 1 of the proof of formula (1.20). Our task in this subsection is to calculate the LHS of (4.3). In our calculations we use the explicit formula (1.12) for the principal symbol $u_{0}^{(j)}(t ; y, \eta)$ which was proved in Section 3.

At $t=0$ formula (3.4) reads

$$
\begin{aligned}
& {\left.\left[\mathfrak{S}_{-1}^{(j)} f_{1}^{(j)}\right]\right|_{t=0}} \\
& \quad=\left.i\left[\frac{\partial^{2}}{\partial x^{\alpha} \eta_{\alpha}}\left(A_{1}(x, \eta)-h^{(j)}(y, \eta)-(x-y)^{\gamma} h_{y^{\nu}}^{(j)}(y, \eta)\right) P^{(j)}(y, \eta)\right]\right|_{x=y}
\end{aligned}
$$

which gives us

$$
\left.\left[\mathfrak{S}_{-1}^{(j)} f_{1}^{(j)}\right]\right|_{t=0}=i\left[\left(A_{1}-h^{(j)}\right)_{y^{\alpha} \eta_{\alpha}} P^{(j)}+\left(A_{1}-h^{(j)}\right)_{y^{\alpha}} P_{\eta_{\alpha}}^{(j)}\right] .
$$

In the latter formula we dropped, for the sake of brevity, the arguments $(y, \eta)$. 
At $t=0$ formula (3.5) reads

$$
\begin{aligned}
& {\left.\left[\mathfrak{S}_{0}^{(j)} f_{0}^{(j)}\right]\right|_{t=0}} \\
& \quad=-i\left\{v^{(j)}, h^{(j)}\right\}\left[v^{(j)}\right]^{*}+\left(A_{0}-q^{(j)}+\frac{i}{2} h_{y^{\alpha} \eta_{\alpha}}^{(j)}\right) P^{(j)}+\left[A_{1}-h^{(j)}\right] u_{-1}^{(j)}(0),
\end{aligned}
$$

where $q^{(j)}$ is the function (1.14) and we dropped, for the sake of brevity, the arguments $(y, \eta)$. Note that in writing down (4.5) we used the fact that

$$
\left.\left[\left(d_{\varphi^{(j)}}\right)^{-1} \partial_{t} d_{\varphi^{(j)}}\right]\right|_{(t, x ; y, \eta)=(0, y ; y, \eta)}=-\frac{1}{2} h_{y^{\alpha} \eta_{\alpha}}^{(j)}(y, \eta)
$$

compare with formula (3.20).

Substituting formulae (4.4) and (4.5) into (4.3) we get

$$
\left(h^{(l)}-h^{(j)}\right) P^{(l)} u_{-1}^{(j)}(0)+P^{(l)} B_{0}^{(j)}=0, \quad l \neq j,
$$

where

$$
B_{0}^{(j)}=\left(A_{0}-q^{(j)}-\frac{i}{2} h_{y^{\alpha} \eta_{\alpha}}^{(j)}+i\left(A_{1}\right)_{y^{\alpha} \eta_{\alpha}}\right) P^{(j)}-i h_{\eta_{\alpha}}^{(j)} P_{y^{\alpha}}^{(j)}+i\left(A_{1}\right)_{y^{\alpha}} P_{\eta_{\alpha}}^{(j)}
$$

The subscript in $B_{0}^{(j)}$ indicates the degree of homogeneity in $\eta$.

4.2. Part 2 of the proof of formula (1.20). Our task in this subsection is to solve the linear algebraic system (4.6) and (4.2) for the unknowns $u_{-1}^{(j)}(0)$.

It is easy to see that the unique solution to the system (4.6) and (4.2) is

$$
u_{-1}^{(j)}(0)=\sum_{l \neq j} \frac{P^{(l)} B_{0}^{(j)}+P^{(j)} B_{0}^{(l)}}{h^{(j)}-h^{(l)}} .
$$

Summation in (4.8) is carried out over all $l$ different from $j$.

4.3. Part 3 of the proof of formula (1.20). Our task in this subsection is to calculate $\left[U^{(j)}(0)\right]_{\text {sub }}$.

We have

$$
\left[U^{(j)}(0)\right]_{\mathrm{sub}}=u_{-1}^{(j)}(0)-\frac{i}{2} P_{y^{\alpha} \eta_{\alpha}}^{(j)}
$$

Here the sign in front of $\frac{i}{2}$ is opposite to that in (1.15) because the way we write $U^{(j)}(0)$ is using the dual symbol. 
Substituting (4.8) and (4.7) into (4.9) we get

$$
\begin{aligned}
& {\left[U^{(j)}(0)\right]_{\text {sub }}} \\
& \qquad-\frac{i}{2} P_{y^{\alpha} \eta_{\alpha}}^{(j)} \\
& \quad+\sum_{l \neq j} \frac{1}{h^{(j)}-h^{(l)}}\left(P ^ { ( l ) } \left[\left(A_{0}+i\left(A_{1}\right)_{\left.\left.y^{\alpha} \eta_{\alpha}\right) P^{(j)}-i h_{\eta_{\alpha}}^{(j)} P_{y^{\alpha}}^{(j)}+i\left(A_{1}\right)_{y^{\alpha}} P_{\eta_{\alpha}}^{(j)}\right]}\right.\right.\right. \\
& \quad+P^{(j)}\left[\left(A_{0}+i\left(A_{1}\right)_{\left.y^{\alpha} \eta_{\alpha}\right) P^{(l)}}\right.\right. \\
& \left.\left.\quad-i h_{\eta_{\alpha}}^{(l)} P_{y^{\alpha}}^{(l)}+i\left(A_{1}\right)_{y^{\alpha}} P_{\eta_{\alpha}}^{(l)}\right]\right) \\
& =\sum_{l \neq j} \frac{P^{(l)} A_{\text {sub }} P^{(j)}+P^{(j)} A_{\text {sub }} P^{(l)}}{h^{(j)}-h^{(l)}}+\frac{i}{2}\left(-P_{y^{\alpha} \eta_{\alpha}}^{(j)}+\sum_{l \neq j} \frac{G_{j l}}{h^{(j)}-h^{(l)}}\right),
\end{aligned}
$$

where

$$
\begin{aligned}
G_{j l} \stackrel{\text { def }}{=} & P^{(l)}\left[\left(A_{1}\right)_{y^{\alpha} \eta_{\alpha}} P^{(j)}-2 h_{\eta_{\alpha}}^{(j)} P_{y^{\alpha}}^{(j)}+2\left(A_{1}\right)_{y^{\alpha}} P_{\eta_{\alpha}}^{(j)}\right] \\
& +P^{(j)}\left[\left(A_{1}\right)_{y}{ }^{\alpha} \eta_{\alpha} P^{(l)}-2 h_{\eta_{\alpha}}^{(l)} P_{y^{\alpha}}^{(l)}+2\left(A_{1}\right)_{y^{\alpha}} P_{\eta_{\alpha}}^{(l)}\right] .
\end{aligned}
$$

We have

$$
\begin{aligned}
& G_{j l}=2 P^{(l)}\left\{A_{1}, P^{(j)}\right\}+2 P^{(j)}\left\{A_{1}, P^{(l)}\right\} \\
& +P^{(l)}\left[\left(A_{1}-h^{(j)}\right)_{y^{\alpha} \eta_{\alpha}} P^{(j)}+2\left(A_{1}-h^{(j)}\right)_{\eta_{\alpha}} P_{y^{\alpha}}^{(j)}\right] \\
& +P^{(j)}\left[\left(A_{1}-h^{(l)}\right)_{y^{\alpha}} \eta_{\alpha} P^{(l)}+2\left(A_{1}-h^{(l)}\right)_{\eta_{\alpha}} P_{y^{\alpha}}^{(l)}\right] \\
& =2 P^{(l)}\left\{A_{1}, P^{(j)}\right\}+2 P^{(j)}\left\{A_{1}, P^{(l)}\right\} \\
& -P^{(l)}\left\{A_{1}-h^{(j)}, P^{(j)}\right\}-P^{(j)}\left\{A_{1}-h^{(l)}, P^{(l)}\right\} \\
& +P^{(l)}\left[\left(A_{1}-h^{(j)}\right)_{y^{\alpha} \eta_{\alpha}} P^{(j)}+\left(A_{1}-h^{(j)}\right)_{\eta_{\alpha}} P_{y^{\alpha}}^{(j)}+\left(A_{1}-h^{(j)}\right)_{y^{\alpha}} P_{\eta_{\alpha}}^{(j)}\right] \\
& +P^{(j)}\left[\left(A_{1}-h^{(l)}\right)_{y^{\alpha} \eta_{\alpha}} P^{(l)}+\left(A_{1}-h^{(l)}\right)_{\eta_{\alpha}} P_{y^{\alpha}}^{(l)}+\left(A_{1}-h^{(l)}\right)_{y^{\alpha}} P_{\eta_{\alpha}}^{(l)}\right] \\
& =P^{(l)}\left\{A_{1}+h^{(j)}, P^{(j)}\right\}+P^{(j)}\left\{A_{1}+h^{(l)}, P^{(l)}\right\} \\
& -P^{(l)}\left(A_{1}-h^{(j)}\right) P_{y^{\alpha} \eta_{\alpha}}^{(j)}-P^{(j)}\left(A_{1}-h^{(l)}\right) P_{y^{\alpha} \eta_{\alpha}}^{(l)} \\
& =P^{(l)}\left\{A_{1}+h^{(j)}, P^{(j)}\right\}+P^{(j)}\left\{A_{1}+h^{(l)}, P^{(l)}\right\} \\
& -P^{(l)}\left(h^{(l)}-h^{(j)}\right) P_{y^{\alpha} \eta_{\alpha}}^{(j)}-P^{(j)}\left(h^{(j)}-h^{(l)}\right) P_{y^{\alpha} \eta_{\alpha}}^{(l)} \\
& =P^{(l)}\left\{A_{1}+h^{(j)}, P^{(j)}\right\}+P^{(j)}\left\{A_{1}+h^{(l)}, P^{(l)}\right\} \\
& +\left(h^{(j)}-h^{(l)}\right)\left(P^{(l)} P_{y^{\alpha} \eta_{\alpha}}^{(j)}-P^{(j)} P_{y^{\alpha} \eta_{\alpha}}^{(l)}\right),
\end{aligned}
$$


so formula (4.10) can be rewritten as

$$
\begin{aligned}
& {\left[U^{(j)}(0)\right]_{\text {sub }}} \\
& =\frac{i}{2}\left(-P_{y^{\alpha} \eta_{\alpha}}^{(j)}+\sum_{l \neq j}\left(P^{(l)} P_{y^{\alpha} \eta_{\alpha}}^{(j)}-P^{(j)} P_{y^{\alpha} \eta_{\alpha}}^{(l)}\right)\right) \\
& \quad+\frac{1}{2} \sum_{l \neq j}\left(\frac{P^{(l)}\left(2 A_{\mathrm{sub}} P^{(j)}+i\left\{A_{1}+h^{(j)}, P^{(j)}\right\}\right)}{h^{(j)}-h^{(l)}}\right. \\
& \left.\quad+\frac{P^{(j)}\left(2 A_{\mathrm{sub}} P^{(l)}+i\left\{A_{1}+h^{(l)}, P^{(l)}\right\}\right)}{h^{(j)}-h^{(l)}}\right) .
\end{aligned}
$$

But

$$
\begin{aligned}
\sum_{l \neq j}\left(P^{(l)} P_{y^{\alpha} \eta_{\alpha}}^{(j)}-P^{(j)} P_{y^{\alpha} \eta_{\alpha}}^{(l)}\right) & =\left(\sum_{l \neq j} P^{(l)}\right) P_{y^{\alpha} \eta_{\alpha}}^{(j)}-P^{(j)}\left(\sum_{l \neq j} P^{(l)}\right)_{y^{\alpha} \eta_{\alpha}} \\
& =\left(I-P^{(j)}\right) P_{y^{\alpha} \eta_{\alpha}}^{(j)}-P^{(j)}\left(I-P^{(j)}\right)_{y^{\alpha} \eta_{\alpha}} \\
& =P_{y^{\alpha} \eta_{\alpha}}^{(j)},
\end{aligned}
$$

so formula (4.11) can be simplified to read

$$
\begin{aligned}
& {\left[U^{(j)}(0)\right]_{\mathrm{sub}}} \\
& \quad=\frac{1}{2} \sum_{l \neq j}\left(\frac{P^{(l)}\left(2 A_{\mathrm{sub}} P^{(j)}+i\left\{A_{1}+h^{(j)}, P^{(j)}\right\}\right)}{h^{(j)}-h^{(l)}}\right. \\
& \left.\quad+\frac{P^{(j)}\left(2 A_{\mathrm{sub}} P^{(l)}+i\left\{A_{1}+h^{(l)}, P^{(l)}\right\}\right)}{h^{(j)}-h^{(l)}}\right) .
\end{aligned}
$$

4.4. Part 4 of the proof of formula (1.20). Our task in this subsection is to calculate $\operatorname{tr}\left[U^{(j)}(0)\right]_{\text {sub }}$.

Formula (4.12) implies

$$
\operatorname{tr}\left[U^{(j)}(0)\right]_{\text {sub }}=\frac{i}{2} \operatorname{tr} \sum_{l \neq j} \frac{P^{(l)}\left\{A_{1}, P^{(j)}\right\}+P^{(j)}\left\{A_{1}, P^{(l)}\right\}}{h^{(j)}-h^{(l)}} .
$$

Put $A_{1}=\sum_{k} h^{(k)} P^{(k)}$ and observe that

- terms with the derivatives of $h$ vanish and

- the only $k$ which may give nonzero contributions are $k=j$ and $k=l$. 
Thus, formula (4.13) becomes

$$
\begin{aligned}
\operatorname{tr}\left[U^{(j)}(0)\right]_{\text {sub }} & \\
=\frac{i}{2} \operatorname{tr} \sum_{l \neq j} \frac{1}{h^{(j)}-h^{(l)}}( & h^{(j)}\left[P^{(l)}\left\{P^{(j)}, P^{(j)}\right\}\right. \\
& \left.+P^{(j)}\left\{P^{(j)}, P^{(l)}\right\}\right]+h^{(l)}\left[P^{(l)}\left\{P^{(l)}, P^{(j)}\right\}\right. \\
& \left.\left.+P^{(j)}\left\{P^{(l)}, P^{(l)}\right\}\right]\right) .
\end{aligned}
$$

We claim that

$$
\begin{aligned}
\operatorname{tr}\left(P^{(l)}\left\{P^{(j)}, P^{(j)}\right\}\right) & =\operatorname{tr}\left(P^{(j)}\left\{P^{(j)}, P^{(l)}\right\}\right) \\
& =-\operatorname{tr}\left(P^{(l)}\left\{P^{(l)}, P^{(j)}\right\}\right) \\
& =-\operatorname{tr}\left(P^{(j)}\left\{P^{(l)}, P^{(l)}\right\}\right) \\
& =\left[v^{(l)}\right]^{*}\left\{v^{(j)},\left[v^{(j)}\right]^{*}\right\} v^{(l)} \\
& =\left(\left[v^{(l)}\right]^{*} v_{y^{\alpha}}^{(j)}\right)\left(\left[v_{\eta_{\alpha}}^{(j)}\right]^{*} v^{(l)}\right)-\left(\left[v^{(l)}\right]^{*} v_{\eta_{\alpha}}^{(j)}\right)\left(\left[v_{y^{\alpha}}^{(j)}\right]^{*} v^{(l)}\right) .
\end{aligned}
$$

These facts are established by writing the orthogonal projections in terms of the eigenvectors and using, if required, the identities

$$
\begin{aligned}
& {\left[v_{y^{\alpha}}^{(l)}\right]^{*} v^{(j)}+\left[v^{(l)}\right]^{*} v_{y^{\alpha}}^{(j)}=0,} \\
& {\left[v_{\eta_{\alpha}}^{(l)}\right]^{*} v^{(j)}+\left[v^{(l)}\right]^{*} v_{\eta_{\alpha}}^{(j)}=0,} \\
& {\left[v_{y^{\alpha}}^{(j)}\right]^{*} v^{(l)}+\left[v^{(j)}\right]^{*} v_{y^{\alpha}}^{(l)}=0,} \\
& {\left[v_{\eta_{\alpha}}^{(j)}\right]^{*} v^{(l)}+\left[v^{(j)}\right]^{*} v_{\eta_{\alpha}}^{(l)}=0 .}
\end{aligned}
$$

In view of the identities (4.15) formula (4.14) can be rewritten as

$$
\begin{aligned}
\operatorname{tr}\left[U^{(j)}(0)\right]_{\mathrm{sub}} & =i \operatorname{tr} \sum_{l \neq j} P^{(l)}\left\{P^{(j)}, P^{(j)}\right\} \\
& =i \operatorname{tr}\left(\left\{P^{(j)}, P^{(j)}\right\}-P^{(j)}\left\{P^{(j)}, P^{(j)}\right\}\right) \\
& =-i \operatorname{tr}\left(P^{(j)}\left\{P^{(j)}, P^{(j)}\right\}\right) .
\end{aligned}
$$


It remains only to simplify the expression in the RHS of (4.16). We have

$$
\begin{aligned}
\operatorname{tr}\left(P^{(j)}\left\{P^{(j)}, P^{(j)}\right\}\right) \\
=\left\{\left[v^{(j)}\right]^{*}, v^{(j)}\right\} \\
\quad+\left[\left(\left[v^{(j)}\right]^{*} v_{y^{\alpha}}^{(j)}\right)\left(\left[v^{(j)}\right]^{*} v_{\eta_{\alpha}}^{(j)}\right)-\left(\left[v^{(j)}\right]^{*} v_{\eta_{\alpha}}^{(j)}\right)\left(\left[v^{(j)}\right]^{*} v_{y^{\alpha}}^{(j)}\right]\right. \\
\quad+\left[\left(\left[v_{y^{\alpha}}^{(j)}\right]^{*} v^{(j)}\right)\left(\left[v_{\eta_{\alpha}}^{(j)}\right]^{*} v^{(j)}\right)-\left(\left[v_{\eta_{\alpha}}^{(j)}\right]^{*} v^{(j)}\right)\left(\left[v_{y^{\alpha}}^{(j)}\right]^{*} v^{(j)}\right)\right] \\
\quad+\left[\left(\left[v^{(j)}\right]^{*} v_{y^{\alpha}}^{(j)}\right)\left(\left[v_{\eta_{\alpha}}^{(j)}\right]^{*} v^{(j)}\right)-\left(\left[v^{(j)}\right]^{*} v_{\eta_{\alpha}}^{(j)}\right)\left(\left[v_{y^{\alpha}}^{(j)}\right]^{*} v^{(j)}\right)\right] \\
=\left\{\left[v^{(j)}\right]^{*}, v^{(j)}\right\}+\left[\left(\left[v^{(j)}\right]^{*} v_{y^{\alpha}}^{(j)}\right)\left(\left[v_{\eta_{\alpha}}^{(j)}\right]^{*} v^{(j)}\right)-\left(\left[v^{(j)}\right]^{*} v_{\eta_{\alpha}}^{(j)}\right)\left(\left[v_{y^{\alpha}}^{(j)}\right]^{*} v^{(j)}\right)\right] \\
=\left\{\left[v^{(j)}\right]^{*}, v^{(j)}\right\}-\left[\left(\left[v^{(j)}\right]^{*} v_{y^{\alpha}}^{(j)}\right)\left(\left[v^{(j)}\right]^{*} v_{\eta_{\alpha}}^{(j)}\right)-\left(\left[v^{(j)}\right]^{*} v_{\eta_{\alpha}}^{(j)}\right)\left(\left[v^{(j)}\right]^{*} v_{y^{\alpha}}^{(j)}\right)\right] \\
=\left\{\left[v^{(j)}\right]^{*}, v^{(j)}\right\} .
\end{aligned}
$$

Formulae (4.16) and (4.17) imply formula (1.20).

\section{U(1) connection}

In the preceding Sections 2-4 we presented technical details of the construction of the propagator. We saw that the eigenvectors of the principal symbol, $v^{(j)}(x, \xi)$, play a major role in this construction. As pointed out in Section 1, each of these eigenvectors is defined up to a U(1) gauge transformation (1.18) and (1.19). In the end, the full symbols (1.11) of our oscillatory integrals $U^{(j)}(t)$ do not depend on the choice of gauge for the eigenvectors $v^{(j)}(x, \xi)$. However, the effect of the gauge transformation (1.18) and (1.19) is not as trivial as it may appear at first sight. We will demonstrate in this section that the gauge transformation (1.18) and (1.19) shows up, in the form of invariantly defined curvature, in the lower order terms $u_{-1}^{(j)}(t ; y, \eta)$ of the symbols of our oscillatory integrals $U^{(j)}(t)$. More precisely, we will show that the RHS of formula (1.20) is the scalar curvature of a connection associated with the gauge transformation (1.18) and (1.19). Further on in this section, until the very last paragraph, the index $j$ enumerating eigenvalues and eigenvectors of the principal symbol is assumed to be fixed.

Consider a smooth curve $\Gamma \subset T^{\prime} M$ connecting points $(y, \eta)$ and $(x, \xi)$. We write this curve in parametric form as $(z(t), \zeta(t)), t \in[0,1]$, so that $(z(0), \zeta(0))=(y, \eta)$ and $(z(1), \zeta(1))=(x, \xi)$. Put

$$
w(t) \stackrel{\text { def }}{=} e^{i \phi(t)} v^{(j)}(z(t), \zeta(t))
$$

where $\phi:[0,1] \rightarrow \mathbb{R}$ is an unknown function which is to be determined from the condition

$$
i w^{*} \dot{w}=0
$$


with the dot indicating the derivative with respect to the parameter $t$. Substituting (5.1) into (5.2) we get an ordinary differential equation for $\phi$ which is easily solved, giving

$$
\begin{aligned}
\phi(1) & =\phi(0)+\int_{0}^{1}\left(\dot{z}^{\alpha}(t) P_{\alpha}(z(t), \zeta(t))+\dot{\zeta}_{\gamma}(t) Q^{\gamma}(z(t), \zeta(t))\right) d t \\
& =\phi(0)+\int_{\Gamma}\left(P_{\alpha} d z^{\alpha}+Q^{\gamma} d \zeta_{\gamma}\right),
\end{aligned}
$$

where

$$
P_{\alpha} \stackrel{\text { def }}{=} i\left[v^{(j)}\right]^{*} v_{z^{\alpha}}^{(j)}, \quad Q^{\gamma} \stackrel{\text { def }}{=} i\left[v^{(j)}\right]^{*} v_{\zeta_{\gamma}}^{(j)} .
$$

Note that the $2 n$-component real quantity $\left(P_{\alpha}, Q^{\gamma}\right)$ is a covector field (1-form) on $T^{\prime} M$. This quantity already appeared in Section 1 as formula (1.21).

Put

$$
\begin{aligned}
& f(y, \eta) \stackrel{\text { def }}{=} e^{i \phi(0)}, \\
& f(x, \xi) \stackrel{\text { def }}{=} e^{i \phi(1)},
\end{aligned}
$$

and rewrite formula (5.3) as

$$
f(x, \xi)=f(y, \eta) e^{i \int_{\Gamma}\left(P_{\alpha} d z^{\alpha}+Q^{\gamma} d \zeta_{\gamma}\right)} .
$$

Let us identify the group $\mathrm{U}(1)$ with the unit circle in the complex plane, i.e. with $f \in \mathbb{C},|f|=1$. We see that formulae (5.5) and (5.4) give us a rule for the parallel transport of elements of the group $\mathrm{U}(1)$ along curves in $T^{\prime} M$. This is the natural $\mathrm{U}(1)$ connection generated by the normalised field of columns of complex-valued scalars

$$
v^{(j)}(z, \zeta)=\left(v_{1}^{(j)}(z, \zeta) \quad \ldots \quad v_{m}^{(j)}(z, \zeta)\right)^{T} .
$$

Recall that the $\Gamma$ appearing in formula (5.5) is a curve connecting points $(y, \eta)$ and $(x, \xi)$, whereas the $v^{(j)}(z, \zeta)$ appearing in formulae (5.4) and (5.6) enters our construction as an eigenvector of the principal symbol of our $m \times m$ matrix pseudodifferential operator $A$.

In practice, dealing with a connection is not as convenient as dealing with the covariant derivative $\nabla$. The covariant derivative corresponding to the connection (5.5) is determined as follows. Let us view the $(x, \xi)$ appearing in formula (5.5) as a variable which takes values close to $(y, \eta)$, and suppose that the curve $\Gamma$ is a short straight (in local coordinates) line segment connecting the point $(y, \eta)$ with the point $(x, \xi)$. We want the covariant derivative of our function $f(x, \xi)$, evaluated at $(y, \eta)$, to be zero. Examination of formula (5.5) shows that the unique covariant derivative satisfying this condition is

$$
\nabla_{\alpha} \stackrel{\text { def }}{=} \partial / \partial x^{\alpha}-i P_{\alpha}(x, \xi) \text { and } \quad \nabla^{\gamma} \stackrel{\text { def }}{=} \partial / \partial \xi_{\gamma}-i Q^{\gamma}(x, \xi) .
$$


We define the curvature of our U(1) connection as

$$
R \stackrel{\text { def }}{=}-i\left(\begin{array}{cc}
\nabla_{\alpha} \nabla_{\beta}-\nabla_{\beta} \nabla_{\alpha} & \nabla_{\alpha} \nabla^{\delta}-\nabla^{\delta} \nabla_{\alpha} \\
\nabla^{\gamma} \nabla_{\beta}-\nabla_{\beta} \nabla^{\gamma} & \nabla^{\gamma} \nabla^{\delta}-\nabla^{\delta} \nabla^{\gamma}
\end{array}\right) .
$$

It may seem that the entries of the $(2 n) \times(2 n)$ matrix (5.8) are differential operators. They are, in fact, operators of multiplication by "scalar functions". Namely, the more explicit form of (5.8) is

$$
R=\left(\begin{array}{cc}
\frac{\partial P_{\alpha}}{\partial x^{\beta}}-\frac{\partial P_{\beta}}{\partial x^{\alpha}} & \frac{\partial P_{\alpha}}{\partial \xi_{\delta}}-\frac{\partial Q^{\delta}}{\partial x^{\alpha}} \\
\frac{\partial Q^{\gamma}}{\partial x^{\beta}}-\frac{\partial P_{\beta}}{\partial \xi_{\gamma}} & \frac{\partial Q^{\gamma}}{\partial \xi_{\delta}}-\frac{\partial Q^{\delta}}{\partial \xi_{\gamma}}
\end{array}\right)
$$

The $(2 n) \times(2 n)$ - component real quantity (5.9) is a rank 2 covariant antisymmetric tensor (2-form) on $T^{\prime} M$. It is an analogue of the electromagnetic tensor.

Substituting (5.4) into (5.9) we get an expression for curvature in terms of the eigenvector of the principal symbol

$$
R=i\left(\begin{array}{ll}
{\left[v_{x^{\beta}}^{(j)}\right]^{*} v_{x^{\alpha}}^{(j)}-\left[v_{x^{\alpha}}^{(j)}\right]^{*} v_{x^{\beta}}^{(j)}} & {\left[v_{\xi_{\delta}}^{(j)}\right]^{*} v_{x^{\alpha}}^{(j)}-\left[v_{x^{\alpha}}^{(j)}\right]^{*} v_{\xi_{\delta}}^{(j)}} \\
{\left[v_{x^{\beta}}^{(j)}\right]^{*} v_{\xi_{\gamma}}^{(j)}-\left[v_{\xi_{\gamma}}^{(j)}\right]^{*} v_{x^{\beta}}^{(j)}} & {\left[v_{\xi_{\delta}}^{(j)}\right]^{*} v_{\xi_{\gamma}}^{(j)}-\left[v_{\xi_{\gamma}}^{(j)}\right]^{*} v_{\xi_{\delta}}^{(j)}}
\end{array}\right)
$$

Examination of formula (5.10) shows that, as expected, curvature is invariant under the gauge transformation (1.18) and (1.19).

It is natural to take the trace of the upper right block in (5.8) which, in the notation (1.16), gives us

$$
-i\left(\nabla_{\alpha} \nabla^{\alpha}-\nabla^{\alpha} \nabla_{\alpha}\right)=-i\left\{\left[v^{(j)}\right]^{*}, v^{(j)}\right\} .
$$

Thus, we have shown that the RHS of formula (1.20) is the scalar curvature of our $\mathrm{U}(1)$ connection.

We end this section by proving, as promised in Section 1, formula (1.22) without referring to microlocal analysis. In the following arguments we use our standard notation for the orthogonal projections onto the eigenspaces of the principal symbol, i.e. we write $P^{(k)} \stackrel{\text { def }}{=} v^{(k)}\left[v^{(k)}\right]^{*}$. We have $\operatorname{tr}\left\{P^{(j)}, P^{(j)}\right\}=0$ and $\sum_{l} P^{(l)}=I$ which implies

$$
\begin{aligned}
0 & =\sum_{l, j} \operatorname{tr}\left(P^{(l)}\left\{P^{(j)}, P^{(j)}\right\}\right) \\
& =\sum_{j} \operatorname{tr}\left(P^{(j)}\left\{P^{(j)}, P^{(j)}\right\}\right)+\sum_{l, j: l \neq j} \operatorname{tr}\left(P^{(l)}\left\{P^{(j)}, P^{(j)}\right\}\right) .
\end{aligned}
$$


But, according to formula (4.15), for $l \neq j$ we have

$$
\operatorname{tr}\left(P^{(l)}\left\{P^{(j)}, P^{(j)}\right\}\right)=-\operatorname{tr}\left(P^{(j)}\left\{P^{(l)}, P^{(l)}\right\}\right),
$$

so the expression in the last sum in the RHS of (5.12) is antisymmetric in the indices $l, j$, which implies that this sum is zero. Hence, formula (5.12) can be rewritten as

$$
\sum_{j} \operatorname{tr}\left(P^{(j)}\left\{P^{(j)}, P^{(j)}\right\}\right)=0 .
$$

It remains only to note that, according to formula (4.17), $\operatorname{tr}\left(P^{(j)}\left\{P^{(j)}, P^{(j)}\right\}\right)=$ $\left\{\left[v^{(j)}\right]^{*}, v^{(j)}\right\}$.

\section{Singularity of the propagator at $t=0$}

Following the notation of [18], we denote by

$$
\mathscr{F}_{\lambda \rightarrow t}[f(\lambda)]=\hat{f}(t)=\int e^{-i t \lambda} f(\lambda) d \lambda
$$

the one-dimensional Fourier transform and by

$$
\mathcal{F}_{t \rightarrow \lambda}^{-1}[\hat{f}(t)]=f(\lambda)=(2 \pi)^{-1} \int e^{i t \lambda} \hat{f}(t) d t
$$

its inverse.

Suppose that we have a Hamiltonian trajectory $\left(x^{(j)}(t ; y, \eta), \xi^{(j)}(t ; y, \eta)\right)$ and a real number $T>0$ such that $x^{(j)}(T ; y, \eta)=y$. We will say in this case that we have a loop of length $T$ originating from the point $y \in M$.

Remark 6.1. There is no need to consider loops of negative length $T$ because, given a $T>0$, we have $x^{(j)}\left(T ; y, \eta^{+}\right)=y$ for some $\eta^{+} \in T_{y}^{\prime} M$ if and only if we have $x^{(j)}\left(-T ; y, \eta^{-}\right)=y$ for some $\eta^{-} \in T_{y}^{\prime} M$. Indeed, it suffices to relate the $\eta^{ \pm}$in accordance with $\eta^{\mp}=\xi^{(j)}\left( \pm T ; y, \eta^{ \pm}\right)$.

Denote by $\mathcal{T}^{(j)} \subset \mathbb{R}$ the set of lengths $T>0$ of all possible loops generated by the Hamiltonian $h^{(j)}$. Here "all possible" refers to all possible starting points $(y, \eta) \in T^{\prime} M$ of Hamiltonian trajectories. It is easy to see that $0 \notin \overline{\mathcal{T}^{(j)}}$. We put

$$
\mathbf{T}^{(j)} \stackrel{\text { def }}{=} \begin{cases}\inf \mathcal{T}^{(j)} & \text { if } \mathcal{T}^{(j)} \neq \emptyset, \\ +\infty & \text { if } \mathcal{T}^{(j)}=\emptyset .\end{cases}
$$


In the Riemannian case (i.e. the case when the Hamiltonian is a square root of a quadratic polynomial in $\xi$ ) it is known, see [14], and [12], that there is a loop originating from every point of the manifold $M$ and, moreover, there is an explicit estimate from above for the number $\mathbf{T}^{(j)}$. We are not aware of similar results for general Hamiltonians.

We also define

$$
\mathbf{T} \stackrel{\text { def }}{=} \min _{j=1, \ldots, m^{+}} \mathbf{T}^{(j)}
$$

Remark 6.2. Note that negative eigenvalues of the principal symbol, i.e. Hamiltonians $h^{(j)}(x, \xi)$ with negative index $j=-1, \ldots,-m^{-}$, do not affect the asymptotic formulae we are about to derive. This is because we are dealing with the case $\lambda \rightarrow+\infty$ rather than $\lambda \rightarrow-\infty$.

Denote by

$$
u(t, x, y) \stackrel{\text { def }}{=} \sum_{k} e^{-i t \lambda_{k}} v_{k}(x)\left[v_{k}(y)\right]^{*}
$$

the integral kernel of the propagator (1.1). The quantity (6.1) can be understood as a distribution in the variable $t \in \mathbb{R}$ depending on the parameters $x, y \in M$.

The main result of this section is the following

Lemma 6.1. Let $\hat{\rho}: \mathbb{R} \rightarrow \mathbb{C}$ be an infinitely smooth function such that

$$
\begin{gathered}
\operatorname{supp} \hat{\rho} \subset(-\mathbf{T}, \mathbf{T}), \\
\hat{\rho}(0)=1, \\
\hat{\rho}^{\prime}(0)=0 .
\end{gathered}
$$

Then, uniformly over $y \in M$, we have

$$
\mathcal{F}_{t \rightarrow \lambda}^{-1}[\hat{\rho}(t) \operatorname{tr} u(t, y, y)]=n a(y) \lambda^{n-1}+(n-1) b(y) \lambda^{n-2}+O\left(\lambda^{n-3}\right)
$$

as $\lambda \rightarrow+\infty$. The densities $a(y)$ and $b(y)$ appearing in the RHS of formula (6.5) are defined in accordance with formulae (1.23) and (1.24).

Proof. Denote by $\left(S_{y}^{*} M\right)^{(j)}$ the $(n-1)$-dimensional unit cosphere in the cotangent fibre defined by the equation $h^{(j)}(y, \eta)=1$ and denote by $d\left(S_{y}^{*} M\right)^{(j)}$ the surface area element on $\left(S_{y}^{*} M\right)^{(j)}$ defined by the condition $d \eta=d\left(S_{y}^{*} M\right)^{(j)} d h^{(j)}$. The latter means that we use spherical coordinates in the cotangent fibre with the Hamiltonian $h^{(j)}$ playing the role of the radial coordinate, see Section 1.1.10 of [18] for details. In particular, as explained in Section 1.1.10 of [18], our surface area element $d\left(S_{y}^{*} M\right)^{(j)}$ is expressed via the Euclidean surface area element as

$$
d\left(S_{y}^{*} M\right)^{(j)}=\left(\sum_{\alpha=1}^{n}\left(h_{\eta_{\alpha}}^{(j)}(y, \eta)\right)^{2}\right)^{-1 / 2} \times \text { Euclidean surface area element. }
$$


Denote also

$$
d\left(S_{y}^{*} M\right)^{(j)} \stackrel{\text { def }}{=}(2 \pi)^{-n} d\left(S_{y}^{*} M\right)^{(j)} .
$$

According to Corollary 4.1.5 from [18] we have uniformly over $y \in M$

$$
\begin{aligned}
\mathscr{F}_{t \rightarrow \lambda}^{-1}[\hat{\rho}(t) \operatorname{tr} u(t, y, y)] \\
=\sum_{j=1}^{m^{+}}\left(c^{(j)}(y) \lambda^{n-1}+d^{(j)}(y) \lambda^{n-2}+e^{(j)}(y) \lambda^{n-2}\right)+O\left(\lambda^{n-3}\right),
\end{aligned}
$$

where

$$
c^{(j)}(y)=\int_{\left(S_{y}^{*} M\right)^{(j)}} \operatorname{tr} u_{0}^{(j)}(0 ; y, \eta) d\left(S_{y}^{*} M\right)^{(j)},
$$

$$
\begin{aligned}
& d^{(j)}(y) \\
& =(n-1) \int_{\left(S_{y}^{*} M\right)^{(j)}} \operatorname{tr}\left(-i \dot{u}_{0}^{(j)}(0 ; y, \eta)\right. \\
& \left.+\frac{i}{2}\left\{\left.u_{0}^{(j)}\right|_{t=0}, h^{(j)}\right\}(y, \eta)\right) d\left(S_{y}^{*} M\right)^{(j)},
\end{aligned}
$$

and

$$
e^{(j)}(y)=\int_{\left(S_{y}^{*} M\right)^{(j)}} \operatorname{tr}\left[U^{(j)}(0)\right]_{\mathrm{sub}}(y, \eta) d\left(S_{y}^{*} M\right)^{(j)} .
$$

In the previous equations, $u_{0}^{(j)}(t ; y, \eta)$ is the principal symbol of the oscillatory integral (2.1) and $\dot{u}_{0}^{(j)}(t ; y, \eta)$ is its time derivative. Note that in writing the term with the Poisson bracket in (6.8) we took account of the fact that Poisson brackets in [18] and in the current paper have opposite signs.

Observe that the integrands in formulae (6.7) and (6.8) are positively homogeneous in $\eta$ of degree 0 , whereas the integrand in formula (6.9) is positively homogeneous in $\eta$ of degree -1 . In order to have the same degree of homogeneity, we rewrite formula (6.9) in equivalent form

$$
e^{(j)}(y)=\int_{\left(S_{y}^{*} M\right)^{(j)}}\left(h^{(j)} \operatorname{tr}\left[U^{(j)}(0)\right]_{\mathrm{sub}}\right)(y, \eta) d\left(S_{y}^{*} M\right)^{(j)} .
$$

Switching from surface integrals to volume integrals with the help of eq. (1.1.15) from [18], we rewrite formulae (6.7) and (6.8) and (6.10) as

$$
c^{(j)}(y)=n \int_{h^{(j)}(y, \eta)<1} \operatorname{tr} u_{0}^{(j)}(0 ; y, \eta) d \eta,
$$




$$
\begin{aligned}
& d^{(j)}(y) \\
& \quad=n(n-1) \int_{h^{(j)}(y, \eta)<1} \operatorname{tr}\left(-i \dot{u}_{0}^{(j)}(0 ; y, \eta)+\frac{i}{2}\left\{\left.u_{0}^{(j)}\right|_{t=0}, h^{(j)}\right\}(y, \eta)\right) d \eta,
\end{aligned}
$$

and

$$
e^{(j)}(y)=n \int_{h^{(j)}(y, \eta)<1}\left(h^{(j)} \operatorname{tr}\left[U^{(j)}(0)\right]_{\mathrm{sub}}\right)(y, \eta) d \eta .
$$

Substituting formulae (1.12) and (1.14) into formulae (6.11) and (6.12) we get

$$
c^{(j)}(y)=n \int_{h^{(j)}(y, \eta)<1} d \eta
$$

and

$$
\begin{aligned}
& d^{(j)}(y) \\
& =-n(n-1) \int_{h^{(j)}(y, \eta)<1}\left(\left[v^{(j)}\right]^{*} A_{\mathrm{sub}} v^{(j)}\right. \\
& \left.\quad-\frac{i}{2}\left\{\left[v^{(j)}\right]^{*}, A_{1}-h^{(j)}, v^{(j)}\right\}\right)(y, \eta) d \eta .
\end{aligned}
$$

Substituting formula (1.20) into formula (6.13) we get

$$
e^{(j)}(y)=-n i \int_{h^{(j)}(y, \eta)<1}\left(h^{(j)}\left\{\left[v^{(j)}\right]^{*}, v^{(j)}\right\}\right)(y, \eta) d \eta .
$$

Substituting formulae (6.14)-(6.16) into formula (6.6) we arrive at (6.5).

Remark 6.3. The proof of Lemma 6.1 given above was based on the use of Corollary 4.1.5 from [18]. In the actual statement of Corollary 4.1.5 in [18] uniformity in $y \in M$ was not mentioned because the authors were dealing with a manifold with a boundary. Uniformity reappeared in the subsequent Theorem 4.2.1 which involved pseudodifferential cut-offs separating the point $y$ from the boundary.

\section{Mollified spectral asymptotics}

Theorem 7.1. Let $\rho: \mathbb{R} \rightarrow \mathbb{C}$ be a function from Schwartz space $S(\mathbb{R})$ whose Fourier transform $\hat{\rho}$ satisfies conditions (6.2)-(6.4). Then, uniformly over $x \in M$, we have

$$
\int e(\lambda-\mu, x, x) \rho(\mu) d \mu=a(x) \lambda^{n}+b(x) \lambda^{n-1}+ \begin{cases}O\left(\lambda^{n-2}\right) & \text { if } n \geq 3, \\ O(\ln \lambda) & \text { if } n=2,\end{cases}
$$


as $\lambda \rightarrow+\infty$. The densities $a(x)$ and $b(x)$ appearing in the RHS of formula (7.1) are defined in accordance with formulae (1.23) and (1.24).

Our spectral function $e(\lambda, x, x)$ was initially defined only for $\lambda>0$, see formula (1.4). We extend the definition to the whole real line by setting

$$
e(\lambda, x, x) \stackrel{\text { def }}{=} 0 \text { for } \lambda \leq 0 .
$$

Proof. Denote by $e^{\prime}(\lambda, x, x)$ the derivative, with respect to the spectral parameter, of the spectral function. Here "derivative" is understood in the sense of distributions. The explicit formula for $e^{\prime}(\lambda, x, x)$ is

$$
e^{\prime}(\lambda, x, x)=\sum_{k=1}^{+\infty}\left\|v_{k}(x)\right\|^{2} \delta\left(\lambda-\lambda_{k}\right) .
$$

Formula (7.2) gives us

$$
\int e^{\prime}(\lambda-\mu, x, x) \rho(\mu) d \mu=\sum_{k=1}^{+\infty}\left\|v_{k}(x)\right\|^{2} \rho\left(\lambda-\lambda_{k}\right) .
$$

Formula (7.3) implies, in particular, that, uniformly over $x \in M$, we have

$$
\int e^{\prime}(\lambda-\mu, x, x) \rho(\mu) d \mu=O\left(|\lambda|^{-\infty}\right) \quad \text { as } \lambda \rightarrow-\infty,
$$

where $O\left(|\lambda|^{-\infty}\right)$ is shorthand for "tends to zero faster than any given inverse power of $|\lambda| "$.

Formula (7.3) can also be rewritten as

$$
\int e^{\prime}(\lambda-\mu, x, x) \rho(\mu) d \mu=\mathcal{F}_{t \rightarrow \lambda}^{-1}[\hat{\rho}(t) \operatorname{tr} u(t, x, x)]-\sum_{k \leq 0}\left\|v_{k}(x)\right\|^{2} \rho\left(\lambda-\lambda_{k}\right),
$$

where the distribution $u(t, x, y)$ is defined in accordance with formula (6.1). Clearly, we have

$$
\sum_{k \leq 0}\left\|v_{k}(x)\right\|^{2} \rho\left(\lambda-\lambda_{k}\right)=O\left(\lambda^{-\infty}\right) \quad \text { as } \lambda \rightarrow+\infty .
$$

Formulae (7.5) and (7.6) and Lemma 6.1 imply that, uniformly over $x \in M$, we have

$$
\begin{aligned}
& \int e^{\prime}(\lambda-\mu, x, x) \rho(\mu) d \mu \\
& \quad=n a(x) \lambda^{n-1}+(n-1) b(x) \lambda^{n-2}+O\left(\lambda^{n-3}\right) \quad \text { as } \lambda \rightarrow+\infty .
\end{aligned}
$$

It remains to note that

$$
\frac{d}{d \lambda} \int e(\lambda-\mu, x, x) \rho(\mu) d \mu=\int e^{\prime}(\lambda-\mu, x, x) \rho(\mu) d \mu .
$$

Formulae (7.8), (7.4), and (7.7) imply (7.1). 
Theorem 7.2. Let $\rho: \mathbb{R} \rightarrow \mathbb{C}$ be a function from Schwartz space $S(\mathbb{R})$ whose Fourier transform $\hat{\rho}$ satisfies conditions (6.2)-(6.4). Then we have

$$
\int N(\lambda-\mu) \rho(\mu) d \mu=a \lambda^{n}+b \lambda^{n-1}+ \begin{cases}O\left(\lambda^{n-2}\right) & \text { if } n \geq 3, \\ O(\ln \lambda) & \text { if } n=2,\end{cases}
$$

as $\lambda \rightarrow+\infty$. The constants $a$ and $b$ appearing in the RHS of formula (7.9) are defined in accordance with formulae (1.8), (1.23), (1.9), and (1.24).

Proof. Formula (7.9) follows from formula (7.1) by integration over $M$, see also formula (1.5).

In stating Theorems 7.1 and 7.2 we assumed the mollifier $\rho$ to be complex-valued. This was done for the sake of generality but may seem unnatural when mollifying real-valued functions $e(\lambda, x, x)$ and $N(\lambda)$. One can make our construction look more natural by dealing only with real-valued mollifiers $\rho$. Note that if the function $\rho$ is real-valued and even then its Fourier transform $\hat{\rho}$ is also real-valued and even and, moreover, condition (6.4) is automatically satisfied.

\section{Unmollified spectral asymptotics}

In this section we derive asymptotic formulae for the spectral function $e(\lambda, x, x)$ and the counting function $N(\lambda)$ without mollification. The section is split into two subsections: in the first we derive one-term asymptotic formulae and in the second two-term asymptotic formulae.

\subsection{One-term spectral asymptotics.}

Theorem 8.1. We have, uniformly over $x \in M$,

$$
e(\lambda, x, x)=a(x) \lambda^{n}+O\left(\lambda^{n-1}\right)
$$

as $\lambda \rightarrow+\infty$.

Proof. The result in question is an immediate consequence of formulae (7.8), (7.7), and Theorem 7.1 from the current paper and Corollary B.2.2 from [18].

Theorem 8.2. We have

$$
N(\lambda)=a \lambda^{n}+O\left(\lambda^{n-1}\right)
$$

as $\lambda \rightarrow+\infty$.

Proof. Formula (8.2) follows from formula (8.1) by integration over $M$, see also formula (1.5). 
8.2. Two-term spectral asymptotics. Up till now, in Section 7 and subsection 8.1, our logic was to derive asymptotic formulae for the spectral function $e(\lambda, x, x)$ first and then obtain corresponding asymptotic formulae for the counting function $N(\lambda)$ by integration over $M$. Such an approach will not work for two-term asymptotics because the geometric conditions required for the existence of two-term asymptotics of $e(\lambda, x, x)$ and $N(\lambda)$ will be different: for $e(\lambda, x, x)$ the appropriate geometric conditions will be formulated in terms of loops, whereas for $N(\lambda)$ the appropriate geometric conditions will be formulated in terms of periodic trajectories.

Hence, in this subsection we deal with the spectral function $e(\lambda, x, x)$ and the counting function $N(\lambda)$ separately.

In what follows the point $y \in M$ is assumed to be fixed.

Denote by $\Pi_{y}^{(j)}$ the set of normalised $\left(h^{(j)}(y, \eta)=1\right)$ covectors $\eta$ which serve as starting points for loops generated by the Hamiltonian $h^{(j)}$. Here "starting point" refers to the starting point of a Hamiltonian trajectory $\left(x^{(j)}(t ; y, \eta), \xi^{(j)}(t ; y, \eta)\right)$ moving forward in time $(t>0)$, see also Remark 6.1.

The reason we are not interested in large negative $t$ is that the refined Fourier Tauberian theorem we will be applying, Theorem B.5.1 from [18], does not require information regarding large negative $t$. And the underlying reason for the latter is the fact that the function we are studying, $e(\lambda, x, x)$ (and, later, $N(\lambda)$ ), is real-valued. The real-valuedness of the function $e(\lambda, x, x)$ implies that its Fourier transform, $\hat{e}(t, x, x)$, possesses the symmetry $\hat{e}(-t, x, x)=\overline{\hat{e}(t, x, x)}$.

The set $\Pi_{y}^{(j)}$ is a subset of the $(n-1)$-dimensional unit cosphere $\left(S_{y}^{*} M\right)^{(j)}$ and the latter is equipped with a natural Lebesgue measure, see proof of Lemma 6.1. It is known, see Lemma 1.8.2 in [18], that the set $\Pi_{y}^{(j)}$ is measurable.

Definition 8.1. A point $y \in M$ is said to be nonfocal if for each $j=1, \ldots, m^{+}$the set $\Pi_{y}^{(j)}$ has measure zero.

With regards to the range of the index $j$ in Definition 8.1, as well as in subsequent Definitions 8.2-8.4, see Remark 6.2.

We call a loop of length $T>0$ absolutely focused if the function

$$
\left|x^{(j)}(T ; y, \eta)-y\right|^{2}
$$

has an infinite order zero in the variable $\eta$, and we denote by $\left(\Pi_{y}^{a}\right)^{(j)}$ the set of normalised $\left(h^{(j)}(y, \eta)=1\right)$ covectors $\eta$ which serve as starting points for absolutely focused loops generated by the Hamiltonian $h^{(j)}$. It is known, see Lemma 1.8.3 in [18], that the set $\left(\Pi_{y}^{a}\right)^{(j)}$ is measurable and, moreover, the set $\Pi_{y}^{(j)} \backslash\left(\Pi_{y}^{a}\right)^{(j)}$ has measure zero. This allows us to reformulate Definition 8.1 as follows.

Definition 8.2. A point $y \in M$ is said to be nonfocal if for each $j=1, \ldots, m^{+}$the set $\left(\Pi_{y}^{a}\right)^{(j)}$ has measure zero. 
In practical applications it is easier to work with Definition 8.2 because the set $\left(\Pi_{y}^{a}\right)^{(j)}$ is usually much thinner than the set $\Pi_{y}^{(j)}$.

In order to derive a two-term asymptotic formula for the spectral function $e(\lambda, x, x)$ we need the following lemma (compare with Lemma 6.1).

Lemma 8.1. Suppose that the point $y \in M$ is nonfocal. Then for any complex-valued function $\hat{\gamma} \in C_{0}^{\infty}(\mathbb{R})$ with supp $\hat{\gamma} \subset(0,+\infty)$ we have

$$
\mathscr{F}_{t \rightarrow \lambda}^{-1}[\hat{\gamma}(t) \operatorname{tr} u(t, y, y)]=o\left(\lambda^{n-1}\right)
$$

as $\lambda \rightarrow+\infty$.

Proof. The result in question is a special case of Theorem 4.4.9 from [18].

The following theorem is our main result regarding the spectral function $e(\lambda, x, x)$.

Theorem 8.3. If the point $x \in M$ is nonfocal then the spectral function $e(\lambda, x, x)$ admits the two-term asymptotic expansion (1.6) as $\lambda \rightarrow+\infty$.

Proof. The result in question is an immediate consequence of formula (7.7), Theorem 7.1 and Lemma 8.1 from the current paper and Theorem B.5.1 from [18].

We now deal with the counting function $N(\lambda)$.

Suppose that we have a Hamiltonian trajectory $\left(x^{(j)}(t ; y, \eta), \xi^{(j)}(t ; y, \eta)\right)$ and a real number $T>0$ such that $\left(x^{(j)}(T ; y, \eta), \xi^{(j)}(T ; y, \eta)\right)=(y, \eta)$. We will say in this case that we have a T-periodic trajectory originating from the point $(y, \eta) \in T^{\prime} M$.

Denote by $\left(S^{*} M\right)^{(j)}$ the unit cosphere bundle, i.e. the $(2 n-1)$-dimensional surface in the cotangent bundle defined by the equation $h^{(j)}(y, \eta)=1$. The unit cosphere bundle is equipped with a natural Lebesgue measure: the $(2 n-1)$-dimensional surface area element on $\left(S^{*} M\right)^{(j)}$ is $d y d\left(S_{y}^{*} M\right)^{(j)}$ where $d\left(S_{y}^{*} M\right)^{(j)}$ is the $(n-1)$-dimensional surface area element on the unit cosphere $\left(S_{y}^{*} M\right)^{(j)}$, see proof of Lemma 6.1.

Denote by $\Pi^{(j)}$ the set of points in $\left(S^{*} M\right)^{(j)}$ which serve as starting points for periodic trajectories generated by the Hamiltonian $h^{(j)}$. It is known, see Lemma 1.3.4 in [18], that the set $\Pi^{(j)}$ is measurable.

Definition 8.3. We say that the nonperiodicity condition is fulfilled if for each $j=$ $1, \ldots, m^{+}$the set $\Pi^{(j)}$ has measure zero.

We call a $T$-periodic trajectory absolutely periodic if the function

$$
\left|x^{(j)}(T ; y, \eta)-y\right|^{2}+\left|\xi^{(j)}(T ; y, \eta)-\eta\right|^{2}
$$

has an infinite order zero in the variables $(y, \eta)$, and we denote by $\left(\Pi^{a}\right)^{(j)}$ the set of points in $\left(S^{*} M\right)^{(j)}$ which serve as starting points for absolutely periodic trajectories 
generated by the Hamiltonian $h^{(j)}$. It is known, see Corollary 1.3.6 in [18], that the set $\left(\Pi^{a}\right)^{(j)}$ is measurable and, moreover, the set $\Pi^{(j)} \backslash\left(\Pi^{a}\right)^{(j)}$ has measure zero. This allows us to reformulate Definition 8.3 as follows.

Definition 8.4. We say that the nonperiodicity condition is fulfilled if for each $j=$ $1, \ldots, m^{+}$the set $\left(\Pi^{a}\right)^{(j)}$ has measure zero.

In practical applications it is easier to work with Definition 8.4 because the set $\left(\Pi^{a}\right)^{(j)}$ is usually much thinner than the set $\Pi^{(j)}$.

In order to derive a two-term asymptotic formula for the counting function $N(\lambda)$ we need the following lemma.

Lemma 8.2. Suppose that the nonperiodicity condition is fulfilled. Then for any complex-valued function $\hat{\gamma} \in C_{0}^{\infty}(\mathbb{R})$ with supp $\hat{\gamma} \subset(0,+\infty)$ we have

$$
\int_{M} \mathcal{F}_{t \rightarrow \lambda}^{-1}[\hat{\gamma}(t) \operatorname{tr} u(t, y, y)] d y=o\left(\lambda^{n-1}\right)
$$

as $\lambda \rightarrow+\infty$.

Proof. The result in question is a special case of Theorem 4.4.1 from [18].

The following theorem is our main result regarding the counting function $N(\lambda)$.

Theorem 8.4. If the nonperiodicity condition is fulfilled then the counting function $N(\lambda)$ admits the two-term asymptotic expansion (1.7) as $\lambda \rightarrow+\infty$.

Proof. The result in question is an immediate consequence of formulae (1.5) and (7.7), Theorem 7.1, and Lemma 8.2 from the current paper and Theorem B.5.1 from [18].

\section{9. $\mathrm{U}(\mathrm{m})$ invariance}

We prove in this section that the RHS of formula (1.24) is invariant under unitary transformations (1.25), and (1.26) of our operator $A$. The arguments presented in this section bear some similarity to those from Section 5, the main difference being that the unitary matrix-function in question is now a function on the base manifold $M$ rather than on $T^{\prime} M$.

Fix a point $x \in M$ and an index $j$ (index enumerating the eigenvalues and eigenvectors of the principal symbol) and consider the expression

$$
\begin{gathered}
\int_{h^{(j)}(x, \xi)<1}\left(\left[v^{(j)}\right]^{*} A_{\mathrm{sub}} v^{(j)}-\frac{i}{2}\left\{\left[v^{(j)}\right]^{*}, A_{1}-h^{(j)}, v^{(j)}\right\}\right. \\
\left.+\frac{i}{n-1} h^{(j)}\left\{\left[v^{(j)}\right]^{*}, v^{(j)}\right\}\right)(x, \xi) d \xi,
\end{gathered}
$$


compare with (1.24). We will show that this expression is invariant under the transformation (1.25) and (1.26).

The transformation (1.25) and (1.26) induces the following transformation of the principal and subprincipal symbols of the operator $A$ :

$$
\begin{aligned}
A_{1} & \longmapsto R A_{1} R^{*}, \\
A_{\text {sub }} & \longmapsto R A_{\text {sub }} R^{*}+\frac{i}{2}\left(R_{x^{\alpha}}\left(A_{1}\right)_{\xi_{\alpha}} R^{*}-R\left(A_{1}\right)_{\xi_{\alpha}} R_{x^{\alpha}}^{*}\right) .
\end{aligned}
$$

The eigenvalues of the principal symbol remain unchanged, whereas the eigenvectors transform as

$$
v^{(j)} \longmapsto R v^{(j)} .
$$

Substituting formulae (9.2)-(9.4) into the RHS of (9.1) we conclude that the increment of the expression (9.1) is

$$
\begin{aligned}
\int_{h^{(j)}(x, \xi)<1}( & \frac{i}{2}\left[v^{(j)}\right]^{*}\left(R^{*} R_{x^{\alpha}}\left(A_{1}\right)_{\xi_{\alpha}}-\left(A_{1}\right)_{\xi_{\alpha}} R_{x^{\alpha}}^{*} R\right) v^{(j)} \\
& -\frac{i}{2}\left(\left[v^{(j)}\right]^{*} R_{x^{\alpha}}^{*} R\left(A_{1}-h^{(j)}\right) v_{\xi_{\alpha}}^{(j)}-\left[v_{\xi_{\alpha}}^{(j)}\right]^{*}\left(A_{1}-h^{(j)}\right) R^{*} R_{x^{\alpha}} v^{(j)}\right) \\
& \left.+\frac{i}{n-1} h^{(j)}\left(\left[v^{(j)}\right]^{*} R_{x^{\alpha}}^{*} R v_{\xi_{\alpha}}^{(j)}-\left[v_{\xi_{\alpha}}^{(j)}\right]^{*} R^{*} R_{x^{\alpha}} v^{(j)}\right)\right)(x, \xi) d \xi
\end{aligned}
$$

which can be rewritten as

$$
\begin{aligned}
-\frac{i}{2} \int_{h^{(j)}(x, \xi)<1} & \left(h_{\xi_{\alpha}}^{(j)}\left(\left[v^{(j)}\right]^{*} R_{x^{\alpha}}^{*} R v^{(j)}-\left[v^{(j)}\right]^{*} R^{*} R_{x^{\alpha}} v^{(j)}\right)\right. \\
& \left.-\frac{2}{n-1} h^{(j)}\left(\left[v^{(j)}\right]^{*} R_{x^{\alpha}}^{*} R v_{\xi_{\alpha}}^{(j)}-\left[v_{\xi_{\alpha}}^{(j)}\right]^{*} R^{*} R_{x^{\alpha}} v^{(j)}\right)\right)(x, \xi) d \xi .
\end{aligned}
$$

In view of the identity $R^{*} R=I$ the above expression can be further simplified, so that it reads now

$$
\begin{aligned}
i \int_{h^{(j)}(x, \xi)<1} & \left(h_{\xi_{\alpha}}^{(j)}\left[v^{(j)}\right]^{*} R^{*} R_{x^{\alpha}} v^{(j)}\right. \\
& \left.\quad-\frac{1}{n-1} h^{(j)}\left(\left[v^{(j)}\right]^{*} R^{*} R_{x^{\alpha}} v_{\xi_{\alpha}}^{(j)}+\left[v_{\xi_{\alpha}}^{(j)}\right]^{*} R^{*} R_{x^{\alpha}} v^{(j)}\right)\right)(x, \xi) d \xi .
\end{aligned}
$$

Denote

$$
B_{\alpha}(x) \stackrel{\text { def }}{=}-i R^{*} R_{x^{\alpha}}
$$

and observe that this set of matrices, enumerated by the tensor index $\alpha$ running through the values $1, \ldots, n$, is Hermitian. Denote also

$$
b_{\alpha}(x, \xi) \stackrel{\text { def }}{=}\left[v^{(j)}\right]^{*} B_{\alpha} v^{(j)}
$$


and observe that these $b_{\alpha}$ are positively homogeneous in $\xi$ of degree 0 . Then the expression (9.5) can be rewritten as

$$
-\int_{h^{(j)}(x, \xi)<1}\left(h_{\xi_{\alpha}}^{(j)} b_{\alpha}-\frac{1}{n-1} h^{(j)} \frac{\partial b_{\alpha}}{\partial \xi_{\alpha}}\right)(x, \xi) d \xi .
$$

Lemma 4.1.4 and formula (1.1.15) from [18] tell us that this expression is zero.

\section{Spectral asymmetry}

In this section we deal with the special case when the operator $A$ is differential (as opposed to pseudodifferential). Our aim is to examine what happens when we change the sign of the operator. In other words, we compare the original operator $A$ with the operator

$$
\widetilde{A} \stackrel{\text { def }}{=}-A \text {. }
$$

In theoretical physics the transformation $A \mapsto-A$ would be interpreted as time reversal, see equation (1.3).

It is easy to see that for a differential operator the number $m$ (number of equations in our system) has to be even and that the principal symbol has to have the same number of positive and negative eigenvalues. In the notation of Section 1 this fact can be expressed as $m=2 m^{+}=2 m^{-}$.

It is also easy to see that the principal symbols of the two operators, $A$ and $\widetilde{A}$, and the eigenvalues and eigenvectors of the principal symbols are related as

$$
\begin{aligned}
A_{1}(x, \xi) & =\tilde{A}_{1}(x,-\xi), \\
h^{(j)}(x, \xi) & =\tilde{h}^{(j)}(x,-\xi),
\end{aligned}
$$

and

$$
v^{(j)}(x, \xi)=\tilde{v}^{(j)}(x,-\xi)
$$

whereas the subprincipal symbols are related as

$$
A_{\text {sub }}(x)=-\tilde{A}_{\text {sub }}(x) .
$$

Formulae (1.23), (1.24), (1.17), (1.16), and (10.1)-(10.4) imply

$$
a(x)=\tilde{a}(x) \quad \text { and } \quad b(x)=-\tilde{b}(x) .
$$

Substituting (10.5) into (1.8) and (1.9) we get

$$
a=\tilde{a} \quad \text { and } \quad b=-\tilde{b} .
$$


Formulae (1.7) and (10.6) imply that the spectrum of a generic first order differential operator is asymmetric about $\lambda=0$. This phenomenon is known as spectral asymmetry, see [1], [2], [3], and [4].

If we square our operator $A$ and consider the spectral problem $A^{2} v=\lambda^{2} v$, then the terms $\pm b \lambda^{n-1}$ cancel out and the second asymptotic coefficient of the counting function (as well as the spectral function) of the operator $A^{2}$ turns to zero. This is in agreement with the known fact that for an even order semi-bounded matrix differential operator acting on a manifold without boundary the second asymptotic coefficient of the counting function is zero, see Section 6 of [20] and [15].

\section{Bibliographic review}

To our knowledge, the first publication on the subject of two-term spectral asymptotics for systems was Ivrii's 1980 paper [6] in Section 2 of which the author stated, without proof, a formula for the second asymptotic coefficient of the counting function. In a subsequent 1982 paper [7] Ivrii acknowledged that the formula from [6] was incorrect and gave a new formula, labelled (0.6), followed by a "proof”. In his 1984 Springer Lecture Notes [8] Ivrii acknowledged on page 226 that both his previous formulae for the second asymptotic coefficient were incorrect and stated, without proof, yet another formula.

Roughly at the same time Rozenblyum [13] also stated a formula for the second asymptotic coefficient of the counting function of a first order system.

The formulae from [6], [7], and [13] are fundamentally flawed because they are proportional to the subprincipal symbol. As our formulae (1.9) and (1.24) show, the second asymptotic coefficient of the counting function may be nonzero even when the subprincipal symbol is zero. This illustrates, yet again, the difference between scalar operators and systems.

The formula on page 226 of [8] gives an algorithm for the calculation of the correction term designed to take account of the effect described in the previous paragraph. This algorithm requires the evaluation of a limit of a complicated expression involving the integral, over the cotangent bundle, of the trace of the symbol of the resolvent of the operator $A$ constructed by means of pseudodifferential calculus. This algorithm was revisited in Ivrii's 1998 book, see formulae (4.3.39) and (4.2.25) in [9].

The next contributor to the subject was Safarov who, in his 1989 DSc Thesis [16], wrote down a formula for the second asymptotic coefficient of the counting function which was "almost" correct. This formula appears in [16] as formula (2.4). As explained in Section 1, Safarov lost only the curvature terms $-\frac{n i}{n-1} \int h^{(j)}\left\{\left[v^{(j)}\right]^{*}, v^{(j)}\right\}$. Safarov's DSc Thesis [16] provides arguments which are sufficiently detailed and we were able to identify the precise point (page 163) at which the mistake occurred.

In 1998 Nicoll rederived [11] Safarov's formula (1.12) for the principal symbols of the propagator, using a method slightly different from [16], but stopped short of calculating the second asymptotic coefficient of the counting function. 
In 2007 Kamotski and Ruzhansky [10] performed an analysis of the propagator of a first order elliptic system based on the approach of Rozenblyum [13], but stopped short of calculating the second asymptotic coefficient of the counting function.

One of the authors of this paper, Vassiliev, considered systems in Section 6 of his 1984 paper [20]. However, that paper dealt with systems of a very special type: differential (as opposed to pseudodifferential) and of even (as opposed to odd) order. In this case the second asymptotic coefficients of the counting function and the spectral function vanish, provided the manifold does not have a boundary.

\section{References}

[1] M. F. Atiyah, V .K. Patodi and I. M. Singer, Spectral asymmetry and Riemannian geometry. Bull. London Math. Soc. 5 (1973), 229-234. MR 0331443 Zbl 0268.58010

[2] M. F. Atiyah, V. K. Patodi and I. M. Singer, Spectral asymmetry and Riemannian geometry I. Math. Proc. Camb. Phil. Soc. 77 (1975), 43-69. MR 0397797 Zbl 0297.58008

[3] M. F. Atiyah, V. K. Patodi and I. M. Singer, Spectral asymmetry and Riemannian geometry II. Math. Proc. Camb. Phil. Soc. 78 (1975), 405-432. MR 0397798 Zbl 0314.58016

[4] M.F. Atiyah, V.K. Patodi and I.M. Singer, Spectral asymmetry and Riemannian geometry III. Math. Proc. Camb. Phil. Soc. 79 (1976), 71-99. MR 0397799 Zbl 0325.58015

[5] J. J. Duistermaat and V. W. Guillemin, The spectrum of positive elliptic operators and periodic bicharacteristics. Invent. Math. 29 (1975), 39-79. MR 0405514 Zbl 0307.35071

[6] V. Ja. Ivrii, On the second term of the spectral asymptotics for the Laplace-Beltrami operator on manifolds with boundary and for elliptic operators acting in fiberings. Dokl. Akad. Nauk SSSR 250 (1980), 1300-1302. English transl. in Sov. Math., Dokl. 21 (1980), 300-302. MR 0564330 Zbl 0448.58024

[7] V. Ja. Ivrii, Accurate spectral asymptotics for elliptic operators that act in vector bundles. Funktsional. Anal. i Prilozhen. 16 (1982), 30-38. English transl. in Funct. Anal. Appl. 16 (1982), 101-108. MR 0659163 Zbl 0549.35100 (original) Zbl 0505.35066 (transl.)

[8] V. Ja. Ivrii, Precise spectral asymptotics for elliptic operators acting in fiberings over manifolds with boundary. Lecture Notes in Mathematics 1100. Springer Verlag, Berlin etc., 1984. MR 0771297 Zbl 0565.35002

[9] V. Ja. Ivrii, Microlocal analysis and precise spectral asymptotics. Springer Monographs in Mathematics. Springer Verlag, Berlin etc., 1998. MR 1631419 Zbl 0906.35003

[10] I. Kamotski and M. Ruzhansky, Regularity properties, representation of solutions, and spectral asymptotics of systems with multiplicities. Comm. Partial Differential Equations 32 (2007), 1-35. MR 2304141 Zbl 1132.35055

[11] W.J. Nicoll, Global oscillatory integrals for solutions of hyperbolic systems. Ph.D. thesis. University of Sussex, Brighton, 1998.

[12] R. Rotman, The length of a shortest geodesic loop at a point. J. Differential Geometry 78 (2008), 497-519. MR 2396252 Zbl 1143.53038 
[13] G. V. Rozenblyum, Spectral asymptotic behavior of elliptic systems. Zap. Nauchn. Sem. Leningrad. Otdel. Mat. Inst. Steklov. (LOMI) 96 (1980), 255-271, 311-312. English transl. in Journal of Mathematical Sciences 21 (1983), 837-850. MR 0579489 Zbl 0507.58045

[14] S. Sabourau, Global and local volume bounds and the shortest geodesic loops. Comm. Anal. Geom. 12 (2004), 1039-1053. MR 2103310 Zbl 1075.53030

[15] Yu. G. Safarov, Exact asymptotics of the spectrum of a boundary value problem, and periodic billiards. Izv. Akad. Nauk SSSR Ser. Mat. 52 (1988), 1230-1251. English transl. in Math. USSR-Izv. 33 (1989), 553-573. MR 0984217 Zbl 0682.35082

[16] Yu. G. Safarov, Non-classical two-term spectral asymptotics for self-adjoint elliptic operators. D.Sc. thesis, Leningrad Branch of the Steklov Mathematical Institute of the USSR Academy of Sciences, Leningrad, 1989.

[17] Yu. G. Safarov, Fourier Tauberian theorems and applications. J. Funct. Anal. 185 (2001), 111-128. MR 1853753 Zbl 1200.35204

[18] Yu. G. Safarov and D. G. Vassiliev, The asymptotic distribution of eigenvalues of partial differential operators. Amer. Math. Soc., Providence, RI, 1997. MR 1414899 Zbl 0870.35003

[19] M. A. Shubin, Pseudodifferential operators and spectral theory. Second ed. Springer Verlag, Berlin, 2001. MR 1852334 Zbl 0980.35180

[20] D. G. Vassiliev, Two-term asymptotics of the spectrum of a boundary value problem under an interior reflection of general form. Funktsional. Anal. i Prilozhen. 18 (1984), 1-13. English trasl. in Funct. Anal. and Appl. 18(1984), 267-277. MR 0775930Zbl 0574.35032

Received April 30, 2012; revised July 25, 2012

Olga Chervova, Department of Mathematics, University College London, Gower Street, London WC1E 6BT, U.K.

E-mail: O.Chervova@ucl.ac.uk

Robert J. Downes, Department of Mathematics, University College London, Gower Street, London WC1E 6BT, U.K.

E-mail: R.Downes@ucl.ac.uk

Dmitri Vassiliev, Department of Mathematics, University College London, Gower Street, London WC1E 6BT, U.K.

E-mail: D.Vassiliev@ucl.ac.uk 